\title{
Meißen oder Wien?
}

\section{Eine framesemantische Analyse von Spracheinstellungen im \\ 18. Jahrhundert}

\author{
Verena Sauer (Kiel)
}

\begin{abstract}
The 18th century was marked by the dispute among scholars which German dialect landscapes should serve as models for the development of Hochdeutsch. In addition to the Upper Saxon variety, which was often mentioned as a prestige variety, also the so called 'Maximilianische Kanzleisprache' of the Viennese Court played an important role in the development of a superior German written language.

The aim of my paper is to reconstruct the historical concept Hochdeutsch by using a framesemantic approach. On the basis of a text corpus that includes 6 grammars of the 18th century, an individual text analysis of the prefaces is carried out. Therefore, I reconstruct explicit predications (so called "fillers" (Ziem 2014:243)) of the reference object Hochdeutsch and then derive the predication potential (so called "slots" (Ziem 2014:243)) of the historical frame Hochdeutsch.
\end{abstract}

\section{Hinführung}

Was ist gutes bzw. richtiges Deutsch? - Die Beantwortung dieser Frage ist zentral im Sprachnormierungsdiskurs des 18. Jahrhunderts. Die Suche nach geeigneten Konzepten des Hochdeutschen sowie die Diskussion darüber trieben die „Sprachkundler“ (Faulstich 2008:1) dieser Zeit in ihren Texten an. Ein Diskurs über das gute und richtige Deutsch entspann sich bereits im 16. Jahrhundert (cf. Josten 1976), u. a. angeregt durch die Humanisten, die Übersetzungen aus dem Lateinischen und anderen Sprachen ins Deutsche anregten, um die Vermittlung von Wissen voranzutreiben (cf. Müller 1969: VII). Darüber hinaus führten die „Ausgleichsbestrebungen“ (ibd.) der Druckereien zu einem vermehrten Verfassen von Orthographielehren, in denen regionale sprachliche Unterschiede erklärt und überregionale Normen empfohlen wurden (cf. ibd.). Die Ausgestaltung einer einheitlichen deutschen Sprache, häufig als Hochdeutsch von den Sprachkundlern bezeichnet (cf. Faulstich 2008:1), wird z. B. von Frangk in seiner 
Orthographia $(1531)^{1}$ oder auch in Kolroß Enchiridion $(1530)^{2}$ thematisiert. Die Spracheinstellungen der Sprachkundler des 16. bis 18. Jahrhunderts spiegeln sich im Sprachnormierungsdiskurs dieser Zeit wieder und sind prägend für die Normierung und schließlich Etablierung des Standarddeutschen im 19. Jahrhundert (cf. von Polenz 1999: 37-76 sowie von Polenz 2009: 125).

Die Konfliktlinien des Sprachnormierungsdiskurses des 18. Jahrhhunderts lassen sich sehr gut anhand der beiden (vermeintlich) orthographischen Varianten <teutsch $>$ und < deutsch> nachvollziehen (cf. Scharloth 2005: 173). Beide Varianten können im Untersuchungskorpus nachgewiesen werden, wobei sich für die ostmitteldeutsche bzw. anomalistische Variante $<$ deutsch $>122$ Belege finden lassen und für die oberdeutsche bzw. analogistische Variante $<$ teutsch> lediglich 89 Belege (siehe hierzu Kapitel 3.3 Datenauswertung). Während die Analogisten die Schreibung <teutsch> für deutsch, also für die Zugehörigkeit zur deutschen Nation verwendeten, schrieben die Anomalisten $<$ deutsch $>.{ }^{3}$ Die Analogisten begründeten ihre Schreibweise mit dem Analogie-Prinzip, also der Ähnlichkeit der Ableitungen. Dementsprechend wäre deutsch von ,deutlich machen, deuten“ (Fulda 1778: 21) und teutsch „vom Teutschen, verteutschen“ (Fulda 1778: 21) abzuleiten. Beide Lexeme müssten folglich unterschieden werden. Fulda bewertete die beiden Varianten im Anschluss sogar explizit, indem er Deutsch „für eine niedere Schreibart, und für eine Neuerung im wahren Hochteutfchen“ (Fulda 1778: 21) beschreibt und seiner Leserschaft rät, „,beym Herkommen zu verbleiben, und Teut $\mathrm{fch}$ zu fchreiben“ (Fulda 1778: 21). Gottsched konstatiert hingegen, „daß man durch alle drey erwaehnte orthographische Regeln ${ }^{4}$ veranlasset wurde, Deutsch und nicht Teutsch zu schreiben“ (Gottsched 1762: 676).

Die erste (sprachideologische) Konfliktlinie, die aus der schulenspezifischen Verwendung von teutsch versus deutsch ersichtlich wird, ist die zwischen Analogisten und Anomalisten. Erstere vertreten die These, dass <teutsch $>$ geschrieben werden müsse, da es von dem Wort „Teutones“ etymologisch hergleitet werden könne. Die Anomalisten schreiben stattdessen $<$ deutsch $>$, weil sie die Schreibung der Aussprache anpassen ${ }^{5}$. Eine zweite (sprachgeographische) Konfliktlinie zeichnet sich zwischen den Anhängern einer ostmitteldeutschen bzw. einer oberdeutschen Leitvarietät ab. Adelung (1781: 16) fasst diesen Disput pointiert zusammen:

\footnotetext{
${ }^{1}$ So schätzt Frangk das Hochdeutsche folgendermaßen ein: „Was nu hie gehandelt oder geschrieben/ wird/ von oberlendischer verstanden. Und wiewol diese sprach an ir selbs rechtfertig und klar/ so ist sie doch in vil puncten vnd stuecken/ auch bey den hochdeutschen nicht einhelich/ Denn sie in keiner jegnit oder lande/ so gantz lauter vnd rein gefurt/ nach gehalden wird/ das nicht weilands etwas straffwirdigs/ oder misbreuchiges darin mitliefft $[\ldots]^{“}$ (Frangk 1531:II).

${ }^{2}$ Kolroß (1530:Aij $\left.{ }^{b}\right)$ wendet sich mit seiner Orthographielehre explizit an die ,hochtüdtschen “:,Dann diß ist fürnnaemlich für die hochtüdtschen gemacht/ würt doch in vylen dingen/ ouch andern tüdtschen nit unnützlich $\sin$ “.

${ }^{3}$ Eine sehr klare und anschauliche Definition von Analogist und Anomalist gibt Scharloth (2005: 175): ,Während der Analogist also die Regelhaftigkeit betont, ist für den Anomalisten der empirisch beobachtbare Sprachgebrauch ausschließliches Kriterium bei der Entscheidung grammatisch strittiger Fragen.“

${ }^{4}$ Als orthographische Regeln unterscheidet Gottsched (1762: 676) nach der Abstammung, der Aussprache und der Gewohnheit.

${ }^{5}$ Gottsched (1762: 680) erklärt diesbezüglich: „Da nun in so vielen Wörtern das Th [...] in neuern Zeiten in ein D verwandelt worden: so ist es ja überaus billig, $[\ldots]$ nicht Teutsch sondern Deutsch zu schreiben.“
} 
Sehr unnütz ist der Streit, ob man diesen Nahmen Deutsch oder Teutsch schreiben müsse. Ganz Nieder-Deutschland schreibt und spricht Düdsch; Ober-Deutschland nach seiner Liebe zu harten Buchstaben Teutsch. Im Hochdeutschen gehet man [...] die Mittelstraße, verbindet beyde, und spricht seit langer Zeit Deutsch.

Adelung (1781: 16)

Daneben formieren sich auch eine sprachsoziologische sowie eine stilistische Konfliktlinie: Es wurde unter den Sprachkundlern jener Zeit debattiert, ob die Bildungseliten bzw. die oberen Schichten oder der Sprachgebrauch der gesamten Bevölkerung Berücksichtigung finden solle, je nachdem ob eher die Zierlichkeit und Klarheit oder die Verständlichkeit als stilistisches Ideal angenommen wurden.

Im Zentrum des vorliegenden Beitrags stehen die Positionen der Sprachkundler des 18. Jahrhunderts, die sich mit einer regionalen Leitvarietät auseinandersetzen. Vor allem der Vorbildcharakter des ostmitteldeutschen Raumes bzw. des oberdeutschen Raumes wurde von den Sprachkundlern heftig diskutiert und soll hier analysiert sowie kritisch reflektiert werden.

Ziel des Beitrages ist es, die historischen Spracheinstellungen bzgl. der Ausprägung einer mitteldeutschen oder oberdeutschen Leitvarietät im 18. Jahrhundert framesemantisch zu rekonstruieren, indem die Einstellungen Aichingers (1754), Antespergs (1749), Bodmers (1768), Dornblüths (1755), Gottscheds (1748) und Popowitschs (1754) gegenübergestellt und miteinander verglichen werden.

In Kapitel 2 des vorliegenden Beitrags wird ein Überblick über das zugrundeliegende Verständnis der Begriffe Konzept, Diskurs und Frame gegeben. Hierfür werden unterschiedliche Definitionsansätze sowie Modellvorstellungen aus der (linguistischen) Forschung vorgestellt und eine eigene Arbeitsdefinition abgeleitet. Dieses theoretische Grundlagenwissen wird in Kapitel 3 empirisch erprobt. Zunächst wird das zugrundeliegende historische Spracheinstellungskorpus dargestellt sowie die einzelnen Schritte der Datenaufbereitung und analyse beschrieben. Im Anschluss wird auf dieser Datenbasis in Kapitel 4 die Frage geklärt: Was ist (Hoch)-Teutsch/-Deutsch? Die Beantwortung erfolgt unter einem sprachgeographischen Fokus. Darauf folgt in Kapitel 5 eine Zusammenfassung der gewonnenen Erkenntnisse sowie ein kurzer Ausblick auf das übergeordnete Forschungsprojekt (cf. Sauer in Vorbereitung).

\section{Konzept - Diskurs - Frame}

Gardt (2017: 2) definiert die Funktion von Diskurs als ,konstitutiv für die intellektuelle Gestaltung des öffentlichen Raumes [...] und damit absolut zentral dafür, wie wir unsere Welt durch und in Sprache erfahren, erschließen und prägen“. Äußerungen, meist Texte, die zu einem Forschungskorpus zusammengestellt werden und die sich mit einem bestimmten diskursimmanenten Thema beschäftigen, dienen hierfür als Basis. Sie spiegeln mehr oder weniger das Wissen bzw. die Einstellungen der Textproduzenten zum Diskursthema wider und ermöglichen die Rekonstruktion der betreffenden Wissenselemente sowie die Durchführung sprachgestützter Analysen (cf. Busse 2018a: 18). Sie sind prägend und handlungsanleitend für den zukünftigen gesellschaftlichen Umgang mit der Thematik (cf. Gardt 2007: 30 sowie weiterführend Gardt 2017 sowie Busse/Teubert 1994). Zudem zeichnen sie sich dadurch aus, dass sie „Regelmäßigkeiten 
im Auftreten bestimmter Wissens-Elemente aufweisen“"(Busse 2018a: 22), da zwischen ihnen häufig intertextuelle Bezüge bestehen. Foucault spricht in diesem Zusammenhang auch von einer Archäologie des Wissens (Foucault 1981), das ist die Ermittlung der Struktur von Wissenselementen im Rahmen von Diskursanalysen (cf. Busse 2018a: 3-4). Diese Wissenselemente zu sprachlichen Varietäten sind ein Bestandteil von Spracheinstellungen.

In der Linguistik werden (Sprach-) Einstellungen meist nach dem Drei-Komponenten-Modell von Rosenberg/Hovland (1966) definert (cf. Scharloth 2005: 6), das diese in ,drei konzeptuell unterscheidbare Reaktionen auf ein bestimmtes Objekt“ (Scharloth 2005: 6) unterteilt, die ,als kognitiv, affektiv und verhaltensbezogen" (Scharloth 2005: 7) näher bestimmt werden können. ${ }^{6}$ Während sich die kognitive Komponente auf das „Wissen über sprachliche Strukturen, auf die Verwendung der Sprache und ihre Funktionen“ (Schröder 2019: 102) bezieht und die affektive Komponente auf die „damit verbundenen Emotionen“ (Schröder 2019: 102), stellt die konative Komponente den ,[potentiellen] Gebrauch oder die [potentielle] Vermeidung von Sprachformen“" (Schröder 2019: 102) in den Fokus (cf. hierzu auch Lenz 2003: 263). ${ }^{7}$ Spracheinstellungen sind darüber hinaus dynamisch. Sie sind ,sprachbiographisch motiviert und stell[en] ein Konstrukt individueller und sozial vermittelter Erfahrungen dar" (Lenz 2003: 271). Das Sprachwissen ist Bestandteil des Alltagswissens, das sich in der frühen Phase der Sozialisation formiert und in Abhängigkeit zu gesellschaftsbedingten, situationsspezifischen und interaktionellen Kontexten entsteht und modifiziert wird (cf. Lenz 2003: 266). In diesem Zusammenhang ist es wichtig, dass (historische) Spracheinstellungen immer hinsichtlich der Gegebenheiten des jeweiligen Kontextes interpretiert werden. Ändert sich dieser Kontext, können sich auch damit in Zusammenhang stehende Spracheinstellungen ändern und sich dynamisch anpassen.

Häufig überlagern sich Einstellungen zu sprachlichen Varietäten mit Einstellungen gegenüber SprecherInnen, die die jeweilige Varietät verwenden bzw. nicht verwenden (cf. Schröder 2019: 103). Stereotype, „empirisch mehr oder weniger fundierte, in der Regel übergeneralisierte $\mathrm{Zu}$ schreibungen [...] [, die] Wissensbestände mit Bewertungen verbinden“ (Schröder 2019: 103) spielen bei der Ausprägung von Spracheinstellungen eine zentrale Rolle. Hierbei handelt es sich um Überzeugungen, die sich auf soziale Gruppen oder Einzelpersonen beziehen und in der gegebenen Gesellschaft sehr weit verbreitet sind (cf. Quasthoff 1998: 48). Schröder (2019: 103) spricht in diesem Zusammenhang auch von ,virulent gesellschaftlich geteilte[n] Konzepte[n]“. Stereotype haben „die logische Form eines Urteils, das in ungerechtfertigt vereinfachender und generalisierender Weise, mit emotional-wertender Tendenz, einer Klasse von Personen bestimmte Eigenschaften oder Verhaltensweisen zu- oder abspricht" (Quasthoff 1998: 48) und ermöglichen es dem Verwender, „Dinge in vorgefertigten, generalisierenden Schablonen zu betrachten“ (Hundt 1992: 7) und so sehr komplexe Sachverhalte überschaubar werden lassen (cf. Hundt 1992: 7).

\footnotetext{
${ }^{6}$,Attitudes are typically defined as predispositions to respond in a particular way towards a specified class of objects. [...] The types of response [...] fall in three major categories: cognitive, affective, and behavioral" (Rosenberg/Hovland 1966: 1).

${ }^{7}$ Lenz spricht im Hinblick auf die konative Komponente von Spracheinstellungen auch von potentiellen Handlungsintentionen, da Spracheinstellungen nicht zwangsläufig zu einer konkreten (Sprach-)Handlung führen müssen, sondern viel mehr als „latente Verhaltensdispositionen interpretiert werden“ (Lenz 2003: 263) sollten.
} 
Dieses stereotype Wissen ist in Frames abgespeichert und wird so ,in seinem Strukturzusammenhang kognitiv abrufbar“" (Ziem 2005: 2). Die Diskursanalyse profitiert dementsprechend von der Framesemantik, einerseits weil sie Frames als Werkzeuge zur Schematisierung und Vereinheitlichung von Diskurselementen nutzen kann und andererseits weil die Framesemantik eine Vergleichbarkeit von Diskursen bzw. Diskursbereichen, etwa auf diachroner Ebene, ermöglicht (cf. Lönneker 2003: 66; Ziem 2008: 283-288).

Frames fungieren folglich als Schemata, „da sie verstehensrelevantes Wissen repräsentieren und strukturieren, das zur Interpretation sprachlicher Ausdrücke herangezogen wird" (Ziem 2008: 257). Ihre Struktur ist sehr gut geeignet, um (historische) Spracheinstellungen bzw. die damit verbundenen gesellschaftlichen Stereotype zu rekonstruieren. Es ist sinnvoll, die Stereotype hinsichtlich der unterschiedlichen Bezugnahmen weiter zu unterteilen. Unter das Autostereotyp, auch Selbstbild genannt, fallen Aussagen darüber, wie ich mich bzw. meine eigene Gruppe wahrnehme (cf. Thiele 2015: 30). Das Heterostereotyp gibt an, wie ich die anderen einschätze oder bewerte (cf. Thiele 2015: 30) und das Metastereotyp basiert ,,auf Annahmen, die innerhalb einer Gruppe (= Ingroup) über eine Fremdgruppe (= Outgroup) und deren Annahmen über sich selbst und andere [...] bestehen" (Thiele 2015: 31). Stereotype können sich sowohl auf Sprecher(-gruppen) als auch auf Objekte und Sachverhalte beziehen (cf. Thiele 2015: 30). Das folgende Textbeispiel aus der Vorrede zu Gottscheds „Sprachkunst“ (1748) soll zur Veranschaulichung von Hetero- und Metastereotyp dienen:

Und hier könnte ich nun meine Vorrede fchließen, wenn nicht, vor kurzem von einem gelehrten Manne, der mitten im Frankenlande gebohren worden, und fich deswegen bloß allein für einen wahrhaften Hochdeutfchen hält; fonft aber feine Ehre in lauter wunderlichen Meynungen fucht, eine feltfame Frage aufgeworfen wäre.

(Gottsched 1748: **5)

Gottsched gibt hier zum einen Auskunft darüber, wie er den Sprachkundler (gemeint ist Dornblüth) aus einer anderen Gruppe (Vertreter der oberdeutschen Leitvarietät) wahrnimmt: Dornblüth ist ein Mann, der „wunderliche Meinungen“ vertritt (cf. Gottsched ibd.) und „gelehrt" (cf. ibd.) ist. Hierbei handelt es sich um das Heterostereotyp Gottscheds zur Person Dornblüth (FE9 ${ }^{9}$ HETEROSTEREOTYP_PERSON_ENGRUPPE). Dieses gibt wieder, wie Gottsched Dornblüth einschätzt bzw. wie er diesen bewertet. Darüber hinaus kann auch ein Metastereotyp im obenstehenden Zitat ermittelt werden: Dornblüth hält sich selbst für den einzig wahren Hochdeutschen (cf. ibd.). Gottsched stellt hier eine Vermutung darüber auf, wie Dornblüth sich selbst sieht und formuliert ein Metastereotyp (FE METASTEREOTYP_PERSON_ENGRUPPE), im Sinne einer Vermutung über die Selbstwahrnehmung einer Person aus der Outgroup (cf. Thiele 2015: 32).

\footnotetext{
${ }^{8}$ Die Beurteilung als ,gelehrt“ stellt kein Kompliment Gottscheds an Dornblüth dar, sondern soll lediglich den Vorwurf, dass Dornblüth (nichtsdestotrotz) sehr kleingeistig sei und veraltete Ansichten vertrete, verstärken.

${ }^{9}$ Die Abkürzung FE steht für Frame-Element (siehe hierzu Tabelle 1).
} 
Die Auto-, Hetero- und Metastereotype, die in den historischen Vorreden enthalten sind, werden in Token-Frames rekonstruiert. Nach Fillmore, dem Begründer des partizipativen FrameNet-Projekts ${ }^{10}$, werden Frames durch Frame-Evozierende Elemente (FEE) bzw. Lexikalische Einheiten (LE) evoziert. Hierbei wird zwischen verschiedenen Frame-Typen, z. B. den Prädikations-Frames und den Konzept-Frames unterschieden (cf. hierzu Ziem 2018: 8-10). Bei den prädikativen Frames, von Busse (2012: 572) auch als „Fillmore-Frames“ bezeichnet, stehen Verben und durch sie vergebene semantische Rollen (sog. Frame-Elemente (FE)) im Zentrum. Nominale Frames, auch als „Barsalou-Frames“ (Busse 2012: 572) bezeichnet, werden hingegen durch Nomen evoziert, deren FE als Attribute zu definieren sind.

Innerhalb des German FrameNets (GFN) werden Frames zunächst zerlegt in einen Zielausdruck, der den Frame evoziert (cf. German FrameNet 2019). Synonym können auch die Termini target, FEE oder $L E$ verwendet werden (cf. German FrameNet 2019). Das FEE eröffnet die FE, die durch Instanzen/Werte gefüllt werden müssen (cf. German FrameNet 2019). Diese Instanzen können nach Ziem (2008: 270) in Form von konkreten Füllwerten (fillers) oder als Standardwerte (default values) auftreten. Sowohl die konkreten Füllwerte, die der aktuellen Wahrnehmung des Individuums entspringen, als auch die Standardwerte, die aus der Erfahrung oder Erinnerung des Individuums inferiert werden, stellen Prädikationen des Referenzobjektes, des FEE dar (cf. Ziem 2008: 286). Welcher Art diese Prädikationen sein können, im Sinne des Prädikationspotentials eines Ausdrucks, wird durch die FE-Struktur des Frames festgelegt (cf. German FrameNet 2019).

D. h., dass das FEE Hochdeutsch den Frame Hochdeutsch evoziert (siehe Abbildung 1), der ein bestimmtes Prädikationspotential aufweist. Dieses Prädikationspotential wird durch die FE im Frame festgelegt, die als Leerstellen fungieren und mit expliziten Füllwerten oder impliziten Standardwerten besetzt werden können. Der Frame könnte etwa das FE ORT umfassen, welches durch den impliziten Standardwert „Hannover" besetzt ist. ${ }^{11}$ Während sich die expliziten Füllwerte auf die aktuelle Wahrnehmung stützen, z. B. eine explizite Prädikation im Text, basieren implizite Standardwerte auf dem (Erfahrungs-)Wissen (z. B. auf Stereotypen) zu einer sprachlichen Referenz, das im Langzeitgedächntnis abgespeichert ist (cf. Ziem 2008: 262). Die Strukturkonstituenten eines Frames nach Ziem (2008: 283-365) bzw. dem GFN sind in Tabelle 1 zusammengefasst und in Abbildung 1 schematisiert:

\footnotetext{
10 Das FrameNet ist ein digitales Lexikon, das Bedeutungen auf Basis von Frames untersucht (cf. Berkeleyer FrameNet (2021)) und über weltweite Tochterprojekte verfügt, wie etwa das Framenet des Deutschen (cf. German FrameNet Projekt (2021)) an der HHU Düsseldorf unter der Leitung von Alexander Ziem.

11 Dieser Sprachmythos, dass in Hannover das reinste Hochdeutsch gesprochen wird, wird u. a. von Elmentaler (2012) thematisiert.
} 


\begin{tabular}{|c|c|c|c|}
\hline Terminus & Synonyme Termini & Erklärung & $\begin{array}{l}\text { notiertes } \\
\text { Beispiel }^{12}\end{array}$ \\
\hline Frame-Name & $\begin{array}{l}\text { Frame-Bezeich- } \\
\text { nung, Frame-Kern }\end{array}$ & 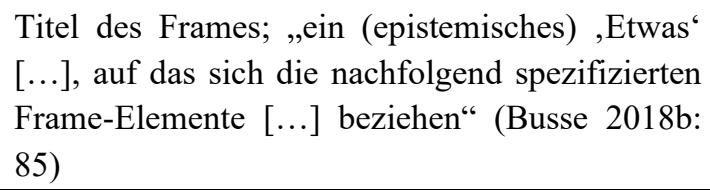 & $\begin{array}{l}\text { Hoch- } \\
\text { deutsch }\end{array}$ \\
\hline $\begin{array}{l}\text { Frame-Evozierendes } \\
\text { Element (FEE) }\end{array}$ & $\begin{array}{l}\text { Target/lexikalische } \\
\text { Einheit/Zielaus- } \\
\text { druck }\end{array}$ & Ausdruck, der einen Frame evoziert & $\begin{array}{l}\text { Hoch- } \\
\text { deutsch }\end{array}$ \\
\hline Frame-Element (FE) & $\begin{array}{l}\text { Slot/Datenkatego- } \\
\text { rie/Leerstelle }\end{array}$ & Frage/Aspekt, die/den der Frame eröffnet & RAUM \\
\hline Wert & Instanz/Phrase & Antwort auf die Frage, die der Frame eröffnet & „Meißen“ \\
\hline $\begin{array}{l}\text { - } \quad \text { konkrete } \\
\text { Füllwerte }\end{array}$ & fillers & $\begin{array}{l}\text { Werte, die sich auf die aktuelle Wahrnehmung } \\
\text { stützen bzw. eine explizite Prädikation im Text }\end{array}$ & \\
\hline $\begin{array}{l}\text { - Standard- } \\
\text { werte }\end{array}$ & default values & $\begin{array}{l}\text { Werte, die im Langzeitgedächtnis abgespeichert } \\
\text { sind und auf Erfahrung oder Erinnerung beruhen }\end{array}$ & \\
\hline
\end{tabular}

Tabelle 1: Strukturkonstituenten von Frames (nach Ziem 2008; German FrameNet 2019)

Die einzelnen Strukturkonstituenten des Frames sind keine isolierten Einheiten, ebenso wenig wie der jeweilige Frame selbst. Jede Instanz (Füllwert/Standardwert) kann zu einem neuen (Sub-)Frame für andere Instanzen werden. Ziem (2008: 270) bezeichnet diese Eigenschaft von Frames als „rekursive Einbettung qua Instantiierung““.

Dies soll anhand des vorhergehenden Beispiels verdeutlicht werden: „Hannover“ ist eine Instanz/ein Füllwert im Hochdeutsch-Frame. „Hannover“ bildet darüber hinaus den (Sub-) Frame Hannover, der über eine eigene FE-Struktur (z. B. FE PERSON_ENGRUPPE) sowie explizite und implizite Füllwerte (z. B. ,protestantische Christengemeinschaft“) verfügt: „Diesen Sachverhalt kann man dahingehend verallgemeinern, dass Schemata ineinander rekursiv eingebettet sind“ (Ziem 2008: 271; cf. auch Barsalou 1992: 43). Instantiierung bedeutet in diesem Zusammenhang, dass einem FE (z. B. PERSON_ENGRUPPE) kategorisch Füllwerte zugeordnet werden, die entweder aus der Erfahrung (Standwerte) abgerufen oder auf Basis konkreter Beobachtungen (konkreter Füllwerte) aufgerufen werden (cf. Ziem 2008: 272; cf. auch Busse 2012: 424). Dynamizität entsteht dann, wenn Füllwerte sehr frequent auftreten und schließlich zu Standardwerten werden, die als Erfahrungswerte aus dem Langzeitgedächtnis abgerufen werden können. Umgekehrt können Standardwerte durch Überschreibung von differenten konkreten Füllwerten ihren Status auch wieder verlieren und aus dem Frame ausgefiltert werden (cf. Busse 2012: 424 sowie Ziem 2008: 272).

Bei der Rekonstruktion von Spracheinstellungen in Konzept-Frames (cf. Busse 2012: 551) stehen Nomen und Adjektive im Zentrum bzw. evozieren als FEE den Frame. Anders als bei prädikativen Frames (cf. Busse 2012: 551), die „eine verbzentrierte Ereignis- bzw. HandlungstypStruktur“ (Busse 2012: 551) aufweisen und über klare prädikative Strukturen (Valenzrahmen)

\footnotetext{
12 Die Strukturkonstituenten des Frames werden wie folgt notiert: Das FEE wird kursiv gesetzt, die FE in Versalien geschrieben und die „Filler“ in Anführungszeichen gesetzt. Die Notation folgt den Konventionen des FrameNets.
} 
verfügen, entsprechen die Frame-Elemente in Konzept-Frames eher „,Attribute[n]' (im Sinne von Eigenschaften)““ (Busse 2012: 572). Im vorliegenden Beitrag stehen Konzept-Frames im Vordergrund sowie deren typische Attribute-Werte-Struktur. Mittels Konzept-Frames als Werkzeuge können die Elemente des historischen Sprachnormierungsdiskurses schematisiert und vereinheitlicht werden.

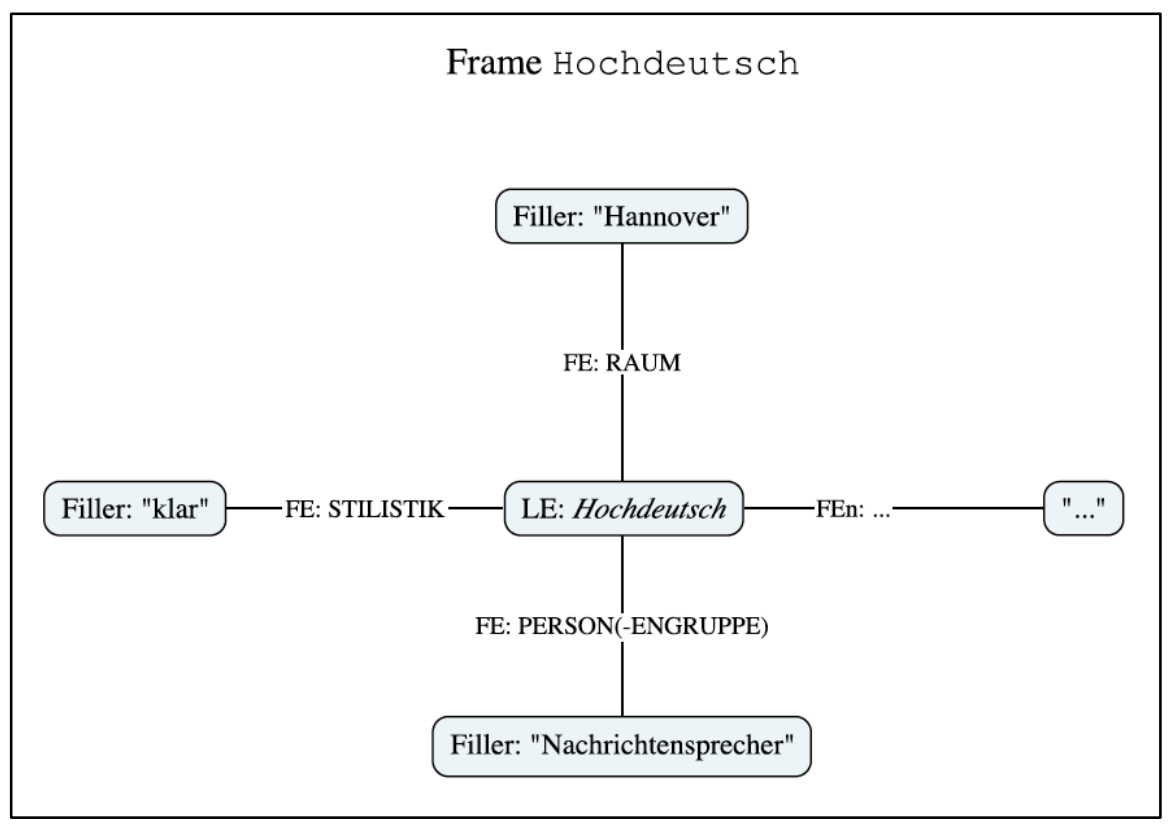

Abbildung 1: Strukturkonstituenten des Frames Hochdeutsch

\section{$3 \quad$ Empirie}

\subsection{Korpuslinguistische Analyse}

Das diesem Beitrag zugrundeliegende Textkorpus umfasst die Vorreden der folgenden historischen Grammatiken des 18. Jahrhunderts:

1. Aichinger (1754): Teutsche Sprachlehre (1065 Word Types/2680 Word Tokens)

2. Antesperg (1749): Kayserlich Deutsche Grammatick (921 Word Types/2015 Word Tokens)

3. Bodmer (1768): Grundsätze der deutschen Sprache (1595 Word Types/4892 Word Tokens)

4. Dornblüth (1755): Observationes (876 Word Types/1963 Word Tokens)

5. Gottsched (1748): Grundlegungen einer Deutschen Sprachkunst (1201 Word Types/3093 Word Tokens)

6. Popowitsch (1754): Anfangsgründe der Teutschen Sprachkunst (2027 Word Types/6274 Word Tokens) 
Die Grammatiken liegen vollständig sowohl als PDF-Datei als auch als TXT-Datei (OCR) vor. ${ }^{13}$ Insgesamt beinhaltet dieses historische Textkorpus (bezogen auf die Vorreden) 4717 Word Types und 20917 Word Tokens.

In einem ersten Schritt mussten die Vorreden im Rahmen einer Autopsie untersucht, d. h. gelesen und erschlossen werden. Die OCR-produzierten Texte waren häufig „schmutzig“, da sie eine bestimmte Anzahl an falscherkannten Zeichen beinhalteten und dementsprechend noch kein zuverlässiges Arbeitsmaterial für eine sprachwissenschaftliche quantitative Analyse bildeten. Erst nach der manuellen Korrektur bzw. Nachbearbeitung konnten die Texte computergestützt weiterbearbeitet werden. Diese manuellen Korrekturen können je nach Verschmutzungsgrad und Zeichenumfang des Textes sehr zeitintensiv sein, weshalb für den vorliegenden Beitrag zunächst nur ein verhältnismäßig kleines Textkorpus als Grundlage dienen kann. ${ }^{14}$ Insgesamt werden die Vorreden jeweils zweimal gelesen, wobei im ersten Durchgang ein Abgleich mit dem Digitalisat des Originals erfolgt und fehlerhafte Zeichen korrigiert werden und im zweiten Durchgang die Inhalte erfasst und Schlüsselwörter herausgefiltert werden. Die korpuslinguistische Analyse der Texte basiert auf den folgenden Verfahren ${ }^{15}$ :

1. Wordlist erstellen: Um die hochfrequenten Wörter (Schlüsselwörter/FEE) im Text erfassen zu können, wird zunächst eine Wordlist erstellt. So ist es möglich, die Anzahl der enthaltenen Word Types und Word Tokens festzustellen und darüber hinaus die Wörter bzw. Lemmata nach ihrer Auftretenshäufigkeit (Frequenz) im Text zu sortieren. Darauf aufbauend werden die zuvor ermittelten Schlüsselwörter/FEE (siehe Tabelle 3) nach ihrer Auftretenshäufigkeit dargestellt. So können hochfrequente Schlüsselwörter bzw. FEE, wie etwa Teutsch/Deutsch herausgefiltert und mit dem Concordance Tool weiterbearbeitet werden.

2. Konkordanzen ermitteln: Die Schlüsselwörter/FEE werden nun als KeyWord In Context (KWIC)-Ansicht dargestellt, sodass deren Kotexte ersichtlich werden. So kann eine Analyse jener Wörter bzw. Wortgruppen (expliziter Füllwerte) durchgeführt werden, die mit dem Schlüsselwort/FEE im Text auftreten. Mit dem Concordance Plot Tool können Schlüsselwörter/FEE sowie deren Kotexte (expliziter Füllwerte) verortet werden, also deren Standort im Text dargestellt werden. Zudem können alle Texte des Korpus hinsichtlich der Standorte des ausgewählten Schlüsselwortes/FEE miteinander verglichen werden. Die Ausgabe der Daten erfolgt im Barcodeformat (siehe Abbildung 2 und Abbildung 3).

\footnotetext{
${ }^{13}$ Das Münchner DigitalisierungsZentrum (MDZ) der Bayerischen Staatsbibliothek stellte mir die Texte als Digitalisate für nichtkommerzielle Zwecke kostenlos über den Downloadservice Daten für die Forschung (DaFo) zur Verfügung.

${ }^{14}$ Das hier in Auszügen vorgestellte Projekt ist Teil des übergeordneten Habilitationsprojektes „Framesemantische Analyse von historischen Spracheinstellungen des 16. bis 18. Jahrhunderts“.

15 Zur Erstellung der Wordlist bzw. zur Ermittlung der Konkordanz wurde die freie Software AntConc verwendet (cf. Anthony 2020).
} 


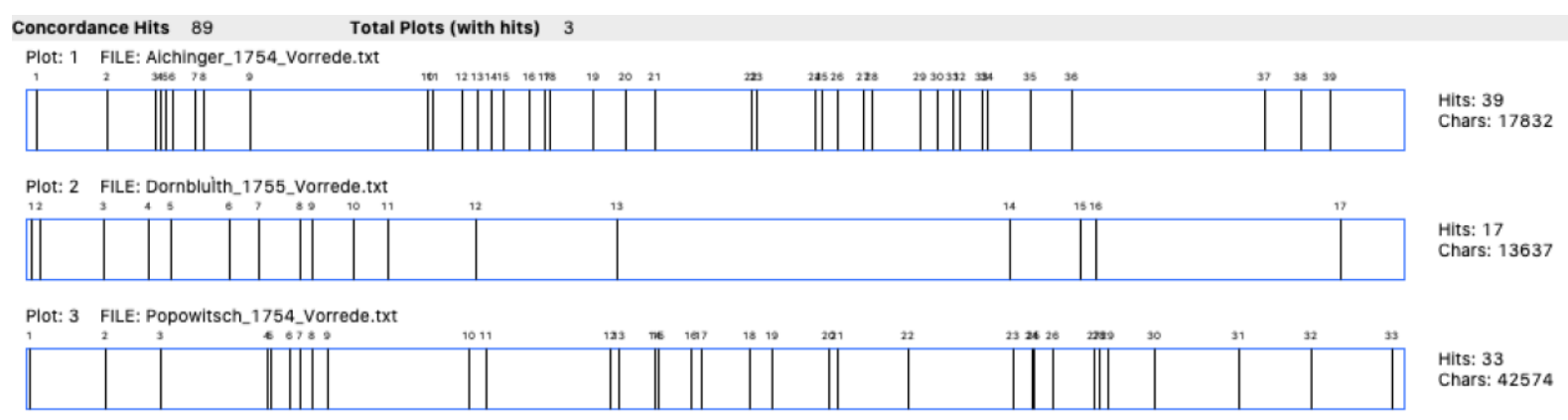

Abbildung 2: Darstellung des Vorkommens des Schlüsselwortes/FEE *teutsch*

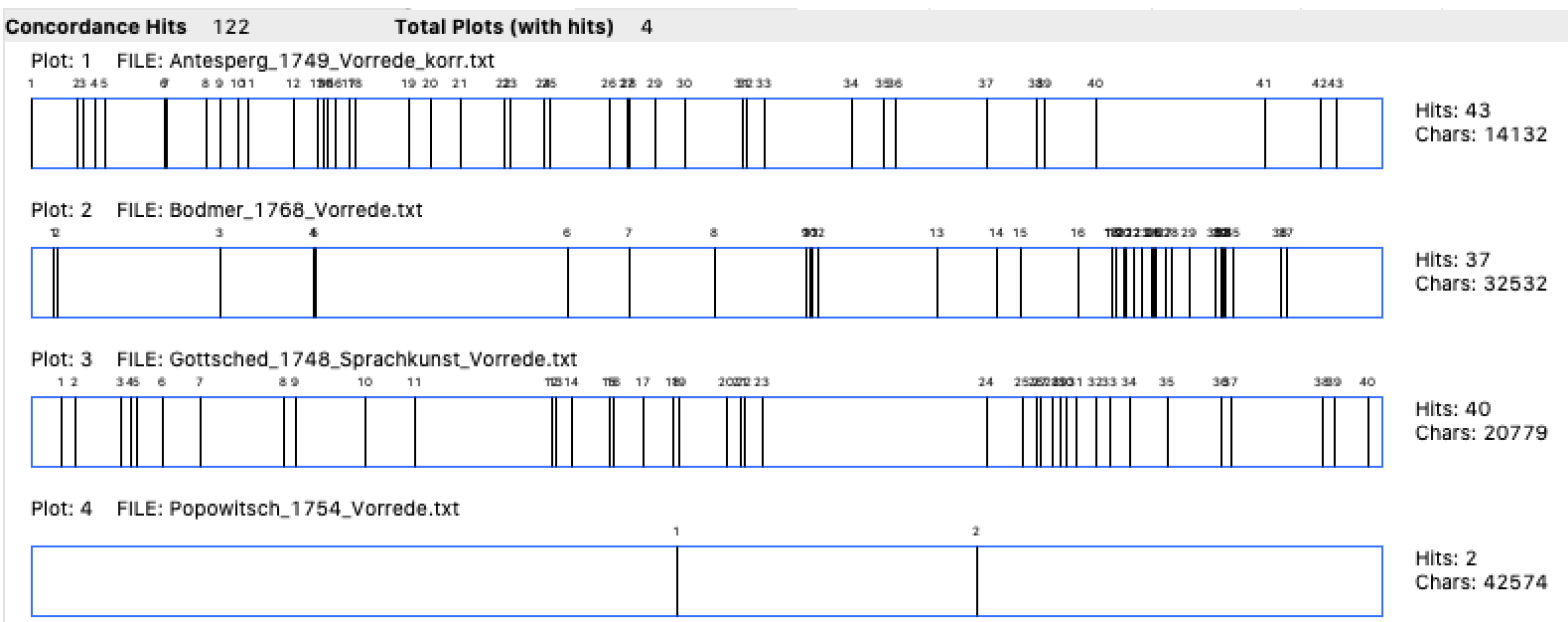

Abbildung 3: Darstellung des Vorkommens des Schlüsselwortes/FEE *deutsch*

Eine umfassende Kenntnis der Inhalte ist dringend erforderlich, um die Ergebnisse aus der quantitativen Analyse sinnvoll interpretieren zu können. Als Beispiel kann die quantitative Häufigkeit des expliziten Füllwerts „Luther“ angeführt werden: In der Vorrede zu den Grundsätzen der deutschen Sprache von Bodmer (1768) ist der explizite Füllwert „Luther“ zwölfmal belegt und tritt hier in einer sehr viel höheren Frequenz auf als in den übrigen analysierten Vorreden. ${ }^{16}$ Die ermittelten Häufigkeiten deuten darauf hin, dass Luther in der Argumentation von Bodmer sehr viel präsenter ist als bei den übrigen Autoren und dementsprechend einen salienten Füllwert in dessen Vorrede markiert. Die qualitative Analyse konnte zeigen, dass Luther in der Vorrede von Bodmer stets als negative Autorität angeführt wird und Argumente erbracht werden, weshalb Luthers Verdienste um die deutsche Sprache nicht so groß waren (cf. Bodmer 1768: 16) wie häufig von anderen Sprachkundlern angenommen. ${ }^{17}$ An diesem Beispiel wird ersichtlich, dass eine rein quantitative Analyse der Spracheinstellungsdaten zwar durchführbar ist, allerdings bei der Interpretation der Ergebnisse u. U. zu Problemen führen kann.

\footnotetext{
16 In den Vorreden von Antesperg (1749), Dornblüth (1755), Gottsched (1748) und Popowitsch (1754) kommt der explizite Füllwert „Luther“ nicht vor, bei Aichinger (1754) ist dieser einmal belegt.

17 „Demnach lernte Luther die Sprache hauptfächlich aus dem Gebrauche und dem Umgange, der, ob er gleich fehr ausgebreitet war doch weit unter der Würde und der Genauigkeit blieb, welche fie von den oben belobten Claffifchen Verfaffern empfangen hatte. Diefes war nun urfache, daß er in diefer verlaffenen Sprache vielfältige Veränderungen vornahm, die ihm die Uneinigkeit im Gebrauche, die Unwiffenheit und Nachlälligkeit, die Vermifchung der alten Redensarten mit neuen, nothwendig zu machen fchienen“ (Bodmer 1768: 16).
} 


\subsection{Framesemantische Analyse}

Die Kotexte zu den FEE (Hoch)-Teutsch/-Deutsch umfassen explizite Füllwerte, anhand derer die FE abgeleitet werden können. Als Beispiel soll eine Belegstelle aus Gottscheds Vorrede zu seiner Sprachkunst (1748) herangezogen werden:

So erlernen fie [die aus entlegenern Provinzen find] denn auch das wahre Hochdeut fche oft beffer, als diejenigen, die zwar mitten im Lande gebohren find.

(Gottsched 1748: **5VII)

Das FEE Hochdeutfche evoziert den Frame Hochdeutsch und weist u. a. die expliziten Füllwerte „fie [die aus entlegenern Provinzen find]“ (Gottsched 1748: **5VII) und „diejenigen, die zwar mitten im Lande gebohren find“" (Gottsched 1748: **5VII) auf. Auf dieser Basis können nun die FE rekonstruiert werden, indem Fragen formuliert werden, auf die diese expliziten Füllwerte eine Antwort geben. Eine sinnvolle Fragestellung wäre etwa: Wer spricht (gutes bzw. schlechteres) Hochdeutsch? Daraus kann das FE PERSON_ENGRUPPE_ALLG_LOK abgeleitet werden. Das FE umfasst dementsprechend jene expliziten Füllwerte im Text, die eine Angabe machen zu regional zuordenbaren Personen(-gruppen), in diesem Beispiel sind es Personen aus entlegneren Provinzen bzw. aus der Mitte des Landes, die (vermeintliches) Hochdeutsch sprechen.

Diese Analyse wurde mit allen historischen Vorreden des Untersuchungskorpus durchgeführt und die folgenden FE, die mit den FEE (Hoch)-Teutsch/-Deutsch vorkommen, rekonstruiert (siehe Tabelle 2):

\begin{tabular}{|c|c|c|}
\hline FE Codes & SUB-FE & Beispielfülllwerte \\
\hline \multirow[t]{3}{*}{ 000_[ORT] } & 001_ORT_ALLG & $\begin{array}{l}\text { „aus entlegenern Provinzen“ (Gottsched } \\
\text { 1748: **5VII) }\end{array}$ \\
\hline & 002_ORT_SPEZ & „,in Wälfchland“ (Gottsched 1748: **5VII) \\
\hline & 003_ORT_AUSBREITUNG & $\begin{array}{l}\text { „mehr als drey hundert deutfche Meilen in } \\
\text { die Länge“ (Gottsched 1748: }{ }^{* *} \text { I) }\end{array}$ \\
\hline \multirow[t]{5}{*}{$\begin{array}{l}\text { 010_[PERSON_EN- } \\
\text { GRUPPE] }\end{array}$} & $\begin{array}{l}\text { 011_PERSON_EN- } \\
\text { GRUPPE_SPEZ }\end{array}$ & „,der Opitzen“ (Gottsched 1748: **5V) \\
\hline & $\begin{array}{l}\text { 012_PERSON_EN- } \\
\text { GRUPPE_ALLG }\end{array}$ & „Leute“ (Gottsched 1748: **I) \\
\hline & $\begin{array}{l}\text { 0121_PERSON_EN- } \\
\text { GRUPPE_ALLG_GE- } \\
\text { SCHLECHT }\end{array}$ & „,gelehrten Männern“ (Gottsched 1748: **2) \\
\hline & $\begin{array}{l}\text { 0122_PERSON_EN- } \\
\text { GRUPPE_ALLG_LOK }\end{array}$ & „,den Deutfchen“ (Gottsched 1748: **5V) \\
\hline & $\begin{array}{l}\text { 0123_PERSON_EN- } \\
\text { GRUPPE_ALLG_BERUF }\end{array}$ & $\begin{array}{l}\text { „bey andern Sprachlehrern“ (Gottsched } \\
1748: * * 3 I)\end{array}$ \\
\hline
\end{tabular}




\begin{tabular}{|c|c|c|}
\hline FE Codes & SUB-FE & Beispielfüllwerte \\
\hline \multirow[t]{4}{*}{ 020_[PRODUKT] } & $\begin{array}{l}\text { 021_PRODUKT_PRODUKTI- } \\
\text { ONSFORM }\end{array}$ & $\begin{array}{l}\text { „im Reden und Schreiben“(Gottsched } \\
1748: * * 3 \mathrm{I})\end{array}$ \\
\hline & $\begin{array}{l}\text { 022_PRODUKT_REZEPTIONS- } \\
\text { FORM }\end{array}$ & „,verfteht“ (Gottsched 1748: **4I) \\
\hline & $\begin{array}{l}\text { 023_PRO- } \\
\text { DUKT_SCHRIFT_ALLG }\end{array}$ & $\begin{array}{l}\text { „die lateinifche Grammatik“ (Gottsched } \\
\text { 1748: **4I) }\end{array}$ \\
\hline & $\begin{array}{l}\text { 024_PRO- } \\
\text { DUKT_SCHRIFT_SPEZ }\end{array}$ & $\begin{array}{l}\text { „,einer Hiftorie der deutfchen Sprachkunft" } \\
\text { (Gottsched 1748: **2I) }\end{array}$ \\
\hline \multirow[t]{3}{*}{ 030_[STILISTIK] } & 031_STILISTIK_ALLG & $\begin{array}{l}\text { „entftellen oder verdunkeln“ (Bodmer 1768: } \\
\text { 22) }\end{array}$ \\
\hline & $\begin{array}{l}\text { 032_STILISTIK_AUSSPRA- } \\
\text { CHE }\end{array}$ & $\begin{array}{l}\text { „regnen mit rechnen, u. f. f. zu verwechfeln“ } \\
\text { (Popowitsch 1754: 19) }\end{array}$ \\
\hline & 033_STILISTIK_SCHREIBUNG & $\begin{array}{l}\text { „Diefe Apoftrophe mag in Umftänden der } \\
\text { Zeiten abgefaffet worden feyn“ (Bodmer } \\
\text { 1768: 24) }\end{array}$ \\
\hline 040_[ZEIT] & - & $\begin{array}{l}\text { „in den Zeiten der Kaifer von dem Schwäbi- } \\
\text { fchen Stamme“ (Bodmer 1768: 8) }\end{array}$ \\
\hline 050_[VARIETÄT] & - & $\begin{array}{l}\text { „Schwäbifche Kaiferliche Hoffprache“ } \\
\text { (Bodmer 1768: 14) }\end{array}$ \\
\hline 060_[APPELL] & - & $\begin{array}{l}\text { „Mundart eines jeden Volkes [...] beurtheilt } \\
\text { werden müffe“ (Aichinger 1754: VII) }\end{array}$ \\
\hline 070_[EREIGNIS] & - & $\begin{array}{l}\text { „fich zu Wien ein vierjähriges Reichshof- } \\
\text { rathliches Interregnum ereignet“ (Antesperg } \\
\text { 1749: §.XIX) }\end{array}$ \\
\hline $\begin{array}{l}\text { 080_[PERSÖNLICH- } \\
\text { KEITSMERKMAL] }\end{array}$ & - & $\begin{array}{l}\text { „fie [Österreicher und Bayern] fchreiben, } \\
\text { wie die Griechen vielfältig nach ihrer eignen } \\
\text { Ausfprache und wollen fich die lieblichere } \\
\text { und reinere Art zu fchreiben nicht aufdrin- } \\
\text { gen laffen“ (Aichinger 1754: XI) }\end{array}$ \\
\hline \multirow[t]{4}{*}{ 090_[BEWERTUNG] } & 091_AUTOSTEREOTYP & $\begin{array}{l}\text { „die Öfterreichifche Mundart von der Hoch- } \\
\text { teutfchen öfters abgehet“ (Popowitsch 1754: } \\
\text { 11) }\end{array}$ \\
\hline & 092_HETEROSTEREOTYP & $\begin{array}{l}\text { „Daß auch die Sachfen, Schlefier und ihre } \\
\text { Nachfolgere im Uberfetzen um kein Haar } \\
\text { gefchickter feyen“ (Dornblüth 1755: 4I) }\end{array}$ \\
\hline & 093_METASTEREOTYP_I & $\begin{array}{l}\text { „,bin also der Meinung nicht, welche zu be- } \\
\text { haubten mir jemand (Gottsched) angeson- } \\
\text { nen hat, dass nicht einerley Sprachlehre } \\
\text { durch ganz Teutschland gelten koenne } \\
\text { (Aichinger 1754: VIII)“ }\end{array}$ \\
\hline & 094_METASTEREOTYP_II & - \\
\hline
\end{tabular}

Tabelle 2: Rekonstrierte FE zum FEE (Hoch)-Teutsch/-Deutsch 
Die individuellen Token-Frames des Untersuchungskorpus beinhalten FE, die Füllwerte zu Orts-, Personen- sowie Produktangaben aufnehmen. Darüber hinaus umfassen die FE Füllwerte, die Angaben machen zur Stilistik, zur Zeit und zur betreffenden Varietät, die mit dem FEE (Hoch)-Teutsch/-Deutsch in Verbindung stehen. Zum Teil können auch FE ermittelt werden, die Angaben zu Appellen, Ereignissen oder Persönlichkeitsmerkmalen enthalten. Hinsichtlich des FE BEWERTUNG spielen Stereotype eine wichtige Rolle. Bei den expliziten Füllwerten, die das FE BEWERTUNG besetzen, handelt es sich immer um Stereotype. Je nachdem, ob sich das Stereotyp (Auto-, Hetero- oder Metastereotyp ${ }^{18}$ ) auf eine Person(-engruppe), eine Varietät oder ein Produkt bezieht, wird das FE z. B. als AUTOSTEREOTYP PERSON_ENGRUPPE $^{19}$, AUTOSTEREOTYP_VARIETÄT ${ }^{20}$ oder AUTOSTEREOTYP_PRODUKT ${ }^{21}$ bezeichnet.

\section{Was ist Hochdeutsch? - Erste Ergebnisse zur sprachgeographischen Leitvarietät im 18. Jahrhundert}

Der Fokus des vorliegenden Beitrages liegt auf den sprachgeographischen Positionen der Sprachkundler des 18. Jahrhunderts. Wie bereits in Kapitel 1 erläutert, weisen die FEE Deutsch und Teutsch eine schulenspezifische Verteilung im Korpus auf: Die mitteldeutsche bzw. anomalistische Variante (Hoch-)Deutsch ist insgesamt 122 mal belegt und wird von Gottsched (40 Belege), aber auch von den oberdeutschen Sprachkundlern Antesperg (43 Belege), Bodmer (37 Belege) und Popowitsch (2 Belege) genutzt (siehe Abbildung 3). Es ist anzunehmen, dass die Verwendung der mitteldeutschen Variante in Antespergs Vorrede auf dessen Austausch mit Gottsched zurückzuführen ist. Antesperg bat Gottsched bei der Erstellung seiner Grammatick (1749) brieflich um Rat, woraufhin Gottsched ihm die Orientierung am ostmitteldeutschen Sprachgebrauch empfahl (Faulstich 2008: 93). Auch der oberdeutsche Sprachkundler Bodmer verwendet die mitteldeutsche Variante Deutsch anstelle der oberdeutschen. Laut Faulstich, die sich auf eine Untersuchung von Schlosser (1985) bezieht, ist diese Widersprüchlichkeit darin begründet, dass sich Bodmer zwar eine alemannische Leitvarietät gewünscht hätte, sich aber durchaus um die Position bzw. die Vorzüge der meißnischen Literatursprache bewusst war und dementsprechend nur wenige Helvetizismen in seinen Schriften nutzte (cf. Faulstich 2008: 104). Die beiden Belegstellen bei Popowitsch sind hingegen nur Zitate des Titels von Gottscheds Grundlegung einer Deutschen Sprachkunst. Bis auf diese zwei Belegstellen verwendet Popowitsch ausschließlich die oberdeutsche Variante Teutsch in seiner Vorrede. Die oberdeutsche bzw. analogistische Variante (Hoch-)Teutsch ist 89 mal im Korpus belegt und wird von

\footnotetext{
${ }^{18}$ Das Metastereotyp I beschreibt ein „[v]ermutetes, vermutetes Autostereotyp“ (Thiele 2015:32) nach dem Schema: So, vermute ich, vermuten die Anderen, würde ich mich sehen. Das Metastereotyp II entspricht hingegen einem „,[v]ermutete[n], vermutete[n] Heterostereotyp“ (Thiele 2015:32) nach dem Schema: So, vermute ich, vermuten die Anderen, würde ich die Anderen sehen.

19 Dieses FE nimmt explizite Füllwerte auf, die Bewertungen des Sprachkundlers von sich selbst bzw. der eigenen Ingroup umfassen.

${ }^{20}$ Dieses FE nimmt explizite Füllwerte auf, die Bewertungen des Sprachkundlers zur seiner eigenen Varietät umfassen.

${ }^{21}$ Dieses FE nimmt explizite Füllwerte auf, die Bewertungen des Sprachkundlers zu seinen eigenen Schriften umfassen.
} 
den oberdeutschen Sprachkundlern Aichinger (39 Belege), Dornblüth (17 Belege) und Popowitsch (33 Belege) gebraucht (siehe Abbildung). Die folgendenden expliziten Füllwerte zum FE ORT konnten aus den Vorreden rekonstruiert werden (siehe Tabelle 3):

\begin{tabular}{|c|c|}
\hline $\begin{array}{l}\text { Types zum FE } \\
\text { ORT }\end{array}$ & Tokens zum FE ORT \\
\hline Deutsch (122) & $\begin{array}{l}\text { „deutsch“/,,Deutsch“, „,deutsche“/,Deutsche“, „,deutschen“/,Deutschen“, „deut- } \\
\text { scher“/,Deutscher“, „,deutsches“/,Deutsches“, „Deutschgeneigter“, „Deutschland“, } \\
\text { „Deutschlandes“, ,hochdeutsche“/, „Hochdeutsche“, „Hochdeutschen“, „,Hofdeutsch“, } \\
\text { „plattdeutsch“, „,undeutsche“, ,,verdeutschen“ }\end{array}$ \\
\hline Teutsch (89) & 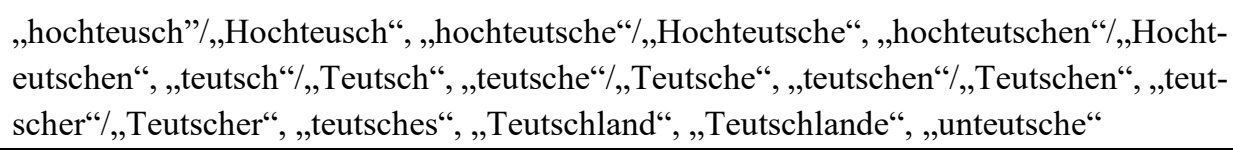 \\
\hline Sächsisch (40) & 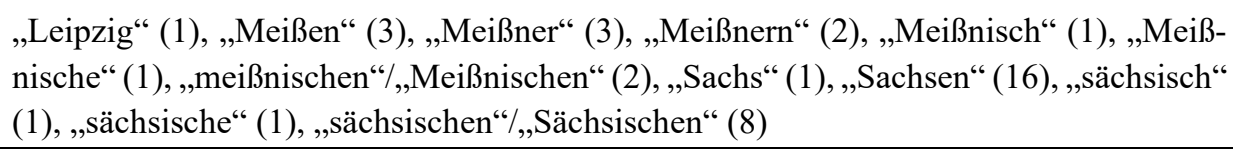 \\
\hline Österreichisch (22) & $\begin{array}{l}\text { „Österreich“ (4), „Österreichern“ (1), „österreichische“ (1), „Osterreichischen“/,,Öster- } \\
\text { reichischen“(4),,,Österreich““(3), ,Osterreicher“ (2), ,Viennensi““(1), „Wien“/,Wienn““ } \\
\text { (4), „wienerisch“/,,wiennerisch“(2) }\end{array}$ \\
\hline Fränkisch (11) & $\begin{array}{l}\text { „altfränkischen“ (1), „, fränkische“/,Fränkische“ (3), „,fränkischen“ (1), „Frankenland“ } \\
\text { (1), „Frankenlande“ (3), Nürnberg (2)) }\end{array}$ \\
\hline Schweizerisch (10) & $\begin{array}{l}\text { „Alpen“ (2), „Bern“ (4), „Schweiz“ (1), „Schweizer“ (1), ,Schweitzerisch“ (1), „,Strass- } \\
\text { burg“ (1) }\end{array}$ \\
\hline Schwäbisch (7) & „Schwab“ (2), „Schwabe“ (1), „Schwäbel“ (1), „Schwäbische“ (1), „Schwäbischen“ (2) \\
\hline Schlesisch (6) & „Schlesier“ (5), „Schlesiern“ (1)), \\
\hline Niedersächsisch (5) & „Niedersachs“ (1), „Niedersachsen“ (4), \\
\hline Pfälzisch (3) & „Oberpfälzische“ (1), „Pfälzer“ (1), „Pfaltz“ (1) \\
\hline Sonstige & $\begin{array}{l}\text { „Bayern“ (1), „Berlin“ (1), „Francfurt“/,Franckfurt“/ „Frankfurt“ (4), „Preußen“ (3), } \\
\text { Preußischem (1), „Rheinländer“ (2), ,,rheinländisch“ (1), „Schleswig“ (1), „Thüringer“ } \\
\text { (1), „thüringischen“ (1), „Westphälinger“ (1) }\end{array}$ \\
\hline
\end{tabular}

\section{Tabelle 3: Types und Tokens zum FE RAUM}

Gottsched war, neben Adelung, der wichtigste Befürworter der meißnischen Leitvarietät und nach dem Erscheinen seiner drei sprachreflektierenden Hauptwerke (Dichtkunst (1729), Redekunst (1736) und Sprachkunst (1748)), „die Autorität im Sprachnormierungsdiskurs“ (Faulstich 2008: 112) des 18. Jahrhunderts. In der Vorrede zur Sprachkunst (1748) gibt Gottsched ${ }^{22}$ zu bedenken, dass das „wahre Hochdeutfche“ (Gottsched 1748: **5VII) studiert und geübt werden müsse (cf. Gottsched 1748: **5VII) und so unter Umständen ,daffelbe in einem plattdeut fchen Munde viel reiner und angenehmer klingt, als in einem fränki chen, thüringifchen und meißnifchen" (Gottsched 1748: **5VII). An dieser Stelle verweist Gottsched u. a. auf die Sprecher aus „entlegenern Provinzen“ (Gottsched 1748: **5VII), die das Hochdeutsche oft besser beherrschen als ,jene, die zwar mitten im Lande gebohren find; fich aber niemals darauf geleget

22 Siehe hierzu die Abbildung 4: Frame Hochdeutsch bei Gottsched: Vorrede zu seiner Sprachkunst (1748) im Anhang. 
haben“ (Gottsched 1748: **5VII). Zwar spricht Gottsched den mitteldeutschen Dialektlandschaften einen sprachgeographischen Vorteil zu, verweist aber dennoch explizit auf die vorhandenen „Provinzialfehler“ (Gottsched 1748: **5VII) im Obersächsischen, die manche „fuer Schoenheiten" (Gottsched 1748: **5VIf.) hielten, die aber durch stetiges Lernen verbessert werden müssten. In diesem Zusammenhang geht Gottsched auf den Sprachgebrauch der oberen Schichten beziehungsweise der Bildungseliten ein, den er als vorbildlich und normkonstituierend ansieht. So empfiehlt er seiner Leserschaft, „fich in dem Umgange mit den Vornehmften und Gelehrteften“ (Gottsched 1748: **5VII) zu üben, um Hochdeutsch besser erlernen zu können. Gottscheds ablehnende Haltung gegenüber der oberdeutschen Varietät kommt ebenfalls in seiner Vorrede zum Ausdruck. Er kritisiert die Haltung Dornblüths scharf, den er nicht namentlich benennt, sondern nur als ,gelehrten Manne“ mit „lauter wunderlichen Meynungen“ (Gottsched 1748: **5) charakterisiert (cf. Blackall 1966: 105-107; cf. von Polenz 2013: 182-183). Gottsched stellt auch Vermutungen zu Dornblüths vermeintlicher Einstellung ,zum alte[n] fraenkische[n] Hofdeutsch“ (Gottsched 1748: **5I) und zu dessen Haltung gegenüber den mitteldeutschen Schreibern, explizit den „Schlesier[n], Meissner[n] und Niedersachsen“ (Gottsched 1748: **5I) an, denen Dornblüth eine ,undeutsche und verderbte Sprache“ (Gottsched 1748: **5I) unterstelle. Die alte fränkische Hofsprache hielte Dornblüth hingegen ,allein fuer recht schoen“ (Gottsched 1748: **5I) und sich selbst ,bloss allein fuer einen wahrhaften Hochdeutschen“ (Gottsched 1748: **5). Gottscheds Spracheinstellung, die auf Basis der Vorrede framesemantisch rekonstruiert werden konnte, umfasst sowohl dessen Autostereotyp als auch Heterostereoytpe und Metastereotype, die auf verschiedene Person(-engruppen) Bezug nehmen (Dornblüth, Plattdeutschsprecher, Mitteldeutsche etc.) bzw. auf das vermutete Autostereotyp ${ }^{23}$ der fremden Person (Dornblüths Haltung zu sich selbst) und das vermutete Heterostereotyp ${ }^{24}$ der fremden Person (Dornblüths Haltung zur fränkischen Hofsprache etc.).

Dornblüth ${ }^{25}$ greift in der Vorrede zu seinen Observationes (1755) Gottsched ebenfalls an. So führt er „,die mehrefte und wichtigfte Fehler der heutigen gemeinen Schreibart“ (Dornblüth 1755: 6f.) darauf zurück, dass ,alle, fonderbar die Ordens-Geiftliche“ (Dornblüth 1755: 6I) den „Herrn Gottfched und denen heutigen Sachfen“ (Dornblüth 1755: 6I) nachahmen. Der große Erfolg von Gottscheds Schriften und dessen diskursdominierende Position sind u. a. auf eben jenen Sachverhalt zurückzuführen, dass er nicht nur eine große Anhängerschaft unter den Gelehrten aus den protestantischen Gegenden Mittel- und Norddeutschlands hatte, sondern auch in den katholisch geprägten Regionen im oberdeutschen Raum. Dornblüth richtet sich in seiner Vorrede sowohl gegen die Vertreter einer obersächsischen Leitvarietät als auch gegen die katholischen Geistlichen, die der Lehre Gottscheds folgten. So bezeichnet er die obersächsische Varietät als ,spoettliche[s] und nichtsheissende[s] Rotwaelsch“ (Dornblüth 1755: 3I), das von den „uebrigen Voelckeren von Teutschland“ (Dornblüth 1755: 3) „fuer gut, schoen, zierlich

\footnotetext{
23 Jürgens definiert das vermutete Autostereotyp als Annahme darüber, „wie andere Gruppen sich selbst sehen“ (Jürgens 2015:68) bzw. als Mutmaßungen, „wie andere über ihren eigenen Dialekt denken“ (Jürgens 2015: 6869).

${ }^{24}$ Das vermutete Heterostereotyp umfasst Annahmen und Mutmaßungen darüber, wie andere Personen(-gruppen) über andere Personen, Varietäten etc. denken und diese bewerten.

${ }^{25}$ Siehe hierzu die Abbildung 5: Frame Hochteutsch bei Dornblüth: Vorrede zu seinen Observationes (1755) im Anhang.
} 
und (wie sie sagen) hochteutsch" (Dornblüth 1755: 3) angenommen würde, sogar von den „höchften teutfchen Cantleyen“ (Dornblüth 1755: 3). Auf seine Haltung zum Hochteutschen geht Dornblüth nur implizit in der Vorrede ein: So könne man eine gute Übersetzung an der „Clarheit und Natürlichkeit ihrer Sprach“ (Dornblüth 1755: 5) erkennen, „weilen die hiftorifche Schreibart unftrittig die leichtefte ift" (Dornblüth 1755: 4I). Sprachgeographisch orientiert sich Dornblüth eher an den oberdeutschen Kanzleien (cf. Faulstich 2008: 122), stellt jedoch ,mit herblichem Bedauren“ (Dornblüth 1755: 3) fest, dass auch diese „dem Sächfifchen Schlender folgen“(Dornblüth 1755: 3I). Während das Autostereotyp Dornblüths nicht explizit in der Vorrede ermittelt werden kann, sind Heterostereotype, u. a. im Hinblick auf die Deutschen bzw. die Sachsen im Text enthalten. Darüber hinaus stellt Dornblüth auch einige Vermutungen zu den Autostereotypen der betreffenden Person(-engruppen) bzw. zu deren Heterostereotypen an. So unterstellt er den „Teutschen“, dass sie nur die fremden bzw. neuen Wörter für schön hielten und als Hochdeutsch annehmen würden (cf. Dornblüth 1755: 3). Auf Basis der von Dornblüth konstruierten Hetero- und Metastereotype können Rückschlüsse auf dessen Autostereotyp gezogen werden ${ }^{26}$ : So kann angenommen werden, dass er ein Verfechter der historischen Schreibart ist und die Verwendung von Fremdwörtern ablehnt, da er moniert, dass die „Teutschen“ (Dornblüth 1755: 2I) alles für schön halten, was „,neu, frembd und nicht gemein ist“ (Dornblüth 1755: 3).

Eine Gegenposition bzgl. des Vorbildanspruchs Meißens bezieht auch Popowitsch (1754) 27 in seiner Sprachkunst, in der er die Stellung des Oberdeutschen, sonderlich der „Osterreichischen Mundart“ (Popowitsch 1754: 11) als Leitvarietät zu verbessern versucht (cf. Faulstich 2008: 126). Auch er richtet sich explizit gegen Gottsched und dessen Sprachkunst, die er als „fuer die Sachsen brauchbarer [...] als fuer uns [Österreicher]“ (Popowitsch 1754: 19) bewertet. Popowitsch benennt in diesem Zusammenhang die zahlreichen Frikativierungen, die in den ostmitteldeutschen Dialekten gehäuft auftreten und die Gottsched in seiner Sprachhkunst ausführlich thematisiert, die für die Österreicher jedoch nicht relevant sind, da man diese ,vergeblich warnen [würde], [...] wagen mit wachen [...] u. s. f. zu verwechseln“ (Popowitsch 1754: 19). Nichtsdestoweniger müsse erkannt werden, dass die Österreichische Mundart „,von der Hochteutschen oefters abgehet" (Popowitsch 1754: 11). Hochdeutsch sieht Popowitsch eher als eine Art Substrat aus allen deutschen Mundarten an (cf. Faulstich 2008: 127). Dementsprechend verurteilt er Gottscheds sprachlandschaftliche Priorisierung des Meißnischen. Gottsched habe im „Wahne gestanden, man muesste, um Hochteutsch schreiben zu koennen, ein gebohrner Sachs oder Schlesier seyn“ (Popowitsch 1754: 31) bis ihn ,die Schweizer seines Irrthums [...] deutlich und gruendlich ueberfuehret" haben. Popowitsch geht hier auf die Rolle Bodmers und Breitingers im spätbarocken Sprachenstreit ein, ohne diese explizit namentlich zu erwähnen. Popowitschs Annahme zum vermuteten Autostereotyp Gottscheds hinsichtlich der Varietät Hochdeutsch stellt ein Metastereotyp dar, das als FE METASTEREOTYP_VARIETÄT eingeordnet wird. Ein weiteres Metastereotyp bezieht sich auf die vermutete Wahrnehmung der

\footnotetext{
26 „Das Heterostereotyp gibt immer Auskunft über das Autostereotyp, denn wenn , die anderen“ so und also ,anders' sind, ergibt sich daraus, wie ,wir" sind“" (Thiele 2015:30).

${ }^{27}$ Siehe hierzu die Abbildung 6: Frame Hochteutsch bei Popowitsch: Vorrede zu seiner Sprachkunst (1754) im Anhang.
} 
„kleinen Geister“. Diese würden einem Menschen, der ,aus keinem Lande gebuertig ist, darinnen die Wissenschaften bluehen“ (Popowitsch 1754: 30) unterstellen, dass jener nicht vernünftig denken könne (cf. Popowitsch 1754: 30). Dieses Metatstereotyp stellt ein vermutetes Heterostereotyp dar und muss dem FE METASTEREOTYP_PERSON(-ENGRUPPE) zugeordnet werden, da es sich auf die Wahrnehmung einer Person (Mensch aus einer bestimmten Region) bezieht und nicht wie im obenstehenden Beispiel auf eine Varietät (Hochteutsch).

Auch Bodmers ${ }^{28}$ Einstellung zu Gottsched und dessen Sprachlehre wandelte sich in der Mitte des 18. Jahrhunderts stark zum Negativen. Während er in der Vorrede zu Breitingers (1740) Dichtkunst die Vorherrschaft des Meißnischen noch anerkennt, stellt er die obersächsische Leitvarietät im Mahler der Sitten (1746) in Frage und wendet sich klar gegen Gottsched (cf. Faulstich 2008: 98). In der Vorrede zu den Grundsätzen der deutschen Sprache hebt Bodmer die „Schwäbifche Kaiferliche Hoffprache“ (Bodmer 1768: 14) besonders hervor sowie die „Minnefinger [und] die Claffifchen Scribenten derfelbigen Sprache“. Luthers Sprache hingegen, dessen „Uneinigkeit im Gebrauche, die Unwiffenheit und Nachläfligkeit, die Vermifchung der alten Redensarten mit neuen“ (Bodmer 1768: 16) verunreinigte die Sprache der „Claffifchen Verfaffer“" (Bodmer 1768: 16) mit „lächerliche[m], platten, altfränkifchen Zeuge“ (Bodmer 1768: 14). Zudem bezeichnet er Luther als ,einen „Gottfchedianer vor Gott Cheden“ (Bodmer 1768: 20), da Luther ebenso wie Gottsched den Sprachgebrauch in das Zentrum seiner Überlegungen stellte und danach übersetzte bzw. schrieb. Bodmer kritisiert, dass „Luther die Sprache hauptfächlich aus dem Gebrauche und dem Umgange [gelernt habe]" (Bodmer 1768: 16), weshalb diese ,einen ftarken Abgang an Wörtern und Ausdrücken erleiden mußte“ (Bodmer 1768: 12). Obgleich Bodmer den Ausdruck Hochdeutsch nicht explizit in seiner Vorrede verwendet ${ }^{29}$, kann sein Autostereotyp rekonstruiert werden: So beurteilt er das Schwäbische der mittelhochdeutschen Minnesänger als „den Genius der Sprache“ (Bodmer 1768: 8). Die „Sprache der Catholifchen Provinzen Deutfchlandes" hebt Bodmer ebenfalls hervor, da diese sich lange Zeit gegen Luther und dessen Sprache aufgelehnt und ,die Sprache der Schwäbifchen Kaifer forgfältiger beybehalten“ (Bodmer 1768: 17) hätten. Die spätere Unterordnung vieler Katholiken bzw. die Anerkennung der „Kunftrichter“ (Bodmer 1768: 24) sowie der ostmitteldeutschen Leitvarietät verurteilte Bodmer hingegen stark.

Antesperg ${ }^{30}$ lehnte die sprachgeographische Bestimmung einer Leitvarietät grundsätzlich ab (cf. Faulstich 2008: 95): ,habe ich mich hierinne nach keiner Mundart [...] gerichtet, fondern nur auf den Grund und auf die von den Gelehrten angenommene deut fche Sprache gefehen“ (Antesperg 1749: §23). Aus dem Zitat kann abgeleitet werden, dass Antesperg den Schriftgebrauch der Gelehrten als Kriterium zur Festsetzung einer überregionalen Schreibnorm ansieht und dementsprechend dem Vorbildanspruch des Meißnischen, wie er von Gottsched propagiert wurde, oder der Erhöhung und Stilisierung des Schwäbischen der mittelhochdeutschen Minnesänger bei Bodmer nicht folgte. Antesperg vertrat stattdessen die These, das die ,regionalen

\footnotetext{
${ }^{28}$ Siehe hierzu die Abbildung 7: Frame Hochdeutsch bei Bodmer: Vorrede zu seinen Grundsätzen der deutschen Sprache (1768) im Anhang.

${ }^{29}$ Bodmer verwendet stattdessen den Ausdruck allgemeiner Geschmack (cf. Bodmer 1768: 8), um sein Konzept einer allgemeinverständlichen und guten deutschen Sprache zu benennen.

${ }^{30}$ Siehe hierzu die Abbildung 8: Frame Hochdeutsch bei Antesperg: Vorrede zu seiner Grammatick (1749) im Anhang.
} 
Schriftsprachen [...] prinzipiell gleichwertig [sind]“" (Faulstich 2008: 95) und beeinflusste damit u. a. Popowitschs Haltung im Sprachnormierungsdiskurs stark (cf. Faulstich 2008: 95). In der Vorrede zu seiner Kayserlich Deutschen Grammatik (1749) verweist er zudem explizit darauf, sowohl „die Approbation der Oefterreichifchen Hofcanzeley“ als auch „der Deutfchen Gefellfchaft in Leipzig" (Antesperg 1749: §20) eingeholt zu haben. Dieser Sachverhalt illustriert sehr gut Antespergs tolerante Haltung und sein Bestreben, die oberdeutsche österreichische Sprache zu verbessern: „So wird die reine deutfche Sprache, Schreibart, Poefie und Beredsamkeit ihre Kinderfchuhe bald vertreten und in Oefterreich zu einem männlichen Alter gelangen" (Antesperg 1749: §33). Antesperg bemängelt u. a. den ,tief eingewurzelte[n] üble[n] Gefchmack in der deutfchen Schreibart, Poefie und Beredfamkeit" (Antesperg 1749: §25), den er durch sein Schaffen verbessern möchte. Diesbezüglich krititsiert er auch die ,lateinifchgelehrte[n] Maenner“ (Antesperg 1749: §3), die „Zwar auf fremde Sprachen fo viel Zeit und Geld“ (Antesperg 1749: §3) anwenden, aber in ihrer deutschen Muttersprache nur „daher lallen“ und „dahin fudlen (Antesperg 1749: §IV). An dieser Stelle eröffnet Antesperg das FE METASTEREOTYP_VARIETÄT, da er im Hinblick auf die Sprechergruppe annimmt, sie wären der Ansicht, dass es ausreichend sei, wenn man die eigene deutsche Muttersprache „mit harter muehe verftehet"، (Antesperg 1749:§IV).

Aichinger ${ }^{31}$ als protestantischer Vertreter aus dem oberdeutschen Raum nahm im Sprachnormierungsdiskurs eine Sonderposition ein, da er sich einerseits durch seine sehr sachliche Position von den anderen, teils sehr polemischen Sprachkundlern (z. B. Dornblüth) unterschied und andererseits die oberdeutsche Kanzleisprachentradition ablehnte (cf. Faulstich 2008: 124-125). So müsse, um die „wahrhafftig hochteutfche Sprache“ (Aichinger 1754: VII) konstruieren zu können, die „Mundart eines jeden Volkes [...] beurtheilt werden“ (Aichinger 1754: VII). Folglich dürften die Meißner ,und etlichen andern Ober- und Niedersachsen, wie [...] Schlesier“ (Aichinger 1754: VIIf.) den Vorzug als sprachgeographische Leitvarietät begehren, müssten diesen aber auch den anderen deutschen Dialektlandschaften zugestehen (cf. Aichinger 1754: VIIf.). Gottsched folge, nach Ansicht Aichingers, ,den Meissnern zuviel“ (Aichinger 1754: IX), „obwohl dieser selbst in Meiffen prouincialifinos, was die Ausfprache belanget“ (Aichinger 1754: VII) finde und eingestehe, dass ,das reine Hochteutfche [...] in keiner Provinz geredet“ (Aichinger 1754: VII) werde. In Bezug auf Gottsched konstruiert Aichinger ein Metastereotyp I im Sinne eines ,vermutete[n], vermutete[n] Autostereotyp[s]“32 (Thiele 2015: 32): So, vermutete Aichinger, vermute Gottsched, dass nach Ansicht Aichingers nur „,einerley Sprachlehre durch ganz Teutschland gelten koenne“ (Aichinger 1754: VIII). Diesen Vorwurf weist Aichinger jedoch explizit von sich. Darüber hinaus kritisiert Aichinger die „Herren Catholicken“ (Aichinger 1754: XI), die „fich nur ums Latein bekümmern“ (Aichinger 1754: XI) und die „Zierlichkeit im Teutfchen“ (Aichinger 1754: XI) nicht sehen bzw. anerkennen wollen.

\footnotetext{
${ }^{31}$ Siehe hierzu die Abbildung 9: Frame Hochteutsch bei Aichinger (1754): Vorrede zu seiner Sprachlehre im Anhang.

32 Das vermutete, vermutete Autostereotyp kann hinsichtlich des obenstehenden Beispiels folgendermaßen umschrieben werden: So, vermutete Aichinger, vermutet Gottsched, würde Aichinger sich sehen.
} 


\section{$5 \quad$ Fazit}

Die eingangs gestellte Frage Was ist gutes bzw. richtiges Deutsch? kann nun bezogen auf die framesemantisch analysierten historischen Vorreden beantwortet werden:

1. Im Mittelpunkt des Sprachnormierungsdiskurses des 18. Jahrhunderts steht die ostmitteldeutsche Leitvarietät, die von Gottsched als wichtigstem Vertreter propagiert wurde. Gottsched ist die zentrale Person im Diskurs, auf den die übrigen Autoren des Untersuchungskorpus explizit ${ }^{33}$ oder implizit ${ }^{34}$ in ihren Vorreden Bezug nehmen.

2. Als Gegendiskurs entspinnt sich eine Diskussion um die (oberdeutschen) Varietäten, die der ostmitteldeutschen Leitvarietät vorzuziehen wären. Im Fokus stehen die „Schwäbifche Kaiferliche Hoffprache“ (Bodmer 1768: 14), die „Osterreichische Mundart“ (Popowitsch 1754: 11) und die Sprache der „höchften teutfchen Cantzleyen“ (Dornblüth 1755: 3).

3. Eine weitere Gegenposition zur ostmitteldeutschen Leitvarietät bezieht Aichinger indem er fordert, dass die „Mundart eines jeden Volkes (...) beurtheilt werden müfle“ (Aichinger 1754: VII).

4. Bezogen auf den Aspekt der Richtigkeit wird von den Sprachkundlern entweder für die Orientierung am Sprachgebrauch (anomalistische Auffassung) oder für ein sprachimmanentes Analogieprinzip (anlogistische Auffassung) plädiert.

Die Korpusdaten konnten zudem belegen, dass Meißen das sprachgeographische Zentrum bildet, jedoch der oberdeutsche bzw. österreichische Raum ebenfalls wichtiger Bestandteil des Sprachnormierungsdiskurses im 18. Jahrhundert ist. So bemühte sich vor allem Antesperg, die reine deutsche Sprache in Österreich zu etablieren, sodass diese dort ,zu einem männlichen Alter gelange“" (Antesperg 1749: §33).

Hinsichtlich der framesemantischen Struktur der Spracheinstellungen kann festgehalten werden, dass es einen Grundstock an FE gibt (siehe Tabelle 2), die diese strukturieren. So konnten die zugrundeliegenden Stereotype innerhalb der Frames rekonstruiert werden. Auffällig ist, dass hinsichtlich des FE VARIETÄT häufig die Werte Sächsisch (40) und Österreichisch (22) auftreten (siehe Tabelle 3). Beide Varietäten bzw. Sprachlandschaften dominieren den Diskurs bezogen auf das Datenmaterial des Untersuchungskorpus. Darüber hinaus fällt bei der Belegung des FE PERSON_ENGRUPPE_SPEZ auf, dass die Füllwerte Gottsched (48) und Luther (15) gehäuft auftreten und somit als diskursdominierende Personen angesehen werden können.

Im vorliegendenden Beitrag stand die sprachgeographische Diskussion innerhalb des Sprachnormierungsdiskurses des 18. Jahrhunderts im Fokus. Das übergeordnete Habilitationsprojekt (cf. Sauer in Vorbereitung) erweitert die Perspektive und rekonstruiert einerseits den Zeitraum vom 16. bis 18. Jahrhunderts und nimmt andererseits auch weitere

\footnotetext{
${ }^{33}$ Explizit verweisen Aichinger (1754), Bodmer (1768), Dornblüth (1755) und Popowitsch (1754) auf Gottsched in ihren Vorreden.

${ }^{34}$ Antesperg (1749) geht auf die „Deutfche Gefellfchaft in Leipzig“ (Antesperg 1749: §. XX) ein und verweist damit implizit auf Gottsched, der von Antesperg das Manuskript zu dessen „Grammatick“ geschickt bekam und durchsah.
} 
Diskussionsschwerpunkte, wie etwa personale und institutionelle Vorbilder sowie stilistische Fragestellungen in den Blick.

Darüber hinaus kann die Entwicklung und Genese regionaler (Gebrauchs-) Standards nachvollzogen werden, die zur Etablierung und Kodifizierung einer allgemeingültigen deutschen Standardorthographie zum Ende des 19. Jahrhunders bzw. zu Beginn des 20. Jahrhunderts ${ }^{35}$ beigetragen haben. Die Ergebnisse des Projekts können dementsprechend auch interessante Hinweise in der aktuellen Debatte zur „Pluriarealität des Standarddeutschen“ (cf. Elspaß/Dürscheid/Ziegler 2017: 21) liefern. So konnten Elspaß/Dürscheid/Ziegler (2017: 5) nachweisen, dass ,viele der als standardsprachlich geltenden Varianten in verschiedenen, z. T. auch nicht zusammenhängenden Gebieten des deutschen Sprachraums vorkommen" und häufig ,mit den alten Dialekträumen übereinstimmen“ (Elspaß/Dürscheid/Ziegler 2017: 4). Die Erkenntnisse zum historischen Spracheinstellungsdiskurs zum Deutschen im 16. bis 18. Jahrhundert sollten zur Interpretation dieses Phänomens unbedingt herangezogen werden.

\section{Literaturverzeichnis}

Adelung, Johann Christoph (1781): Über die Geschichte der Deutschen Sprache, über Deutsche Mundarten und Deutsche Sprachlehre. Leipzig: Breitkopf.

Aichinger, Carl Friedrich (1754): Versuch einer teutschen Sprachlehre, anfänglich nur zu eignem Gebrauche unternommen, endlich aber, um den Gelehrten zu fernerer Untersuchung Anlaß zu geben, ans Liecht gestellt. Frankfurt/Leipzig: Kraus.

Antesperg, Johann Balthasar von (1749): Die kayserliche deutsche Grammatick, oder Kunst die deutsche Sprache recht zu reden, und ohne Fehler zu schreiben in vier Theilen mit einem Examine und zulänglichen Vor- und Anmerkungen, zum Nutzen des gemeinen Wesens, und deren, welche des regelmäßigen Verstandes und reines Ausdruckes in eigener Sprache mächtig seyn wollen, oder ihres Amtes und ihrer Geschäfte halber. Wien: Heninger.

Anthony, Laurence (2020): AntConc (Version 3.5.9) [Computer Software]. Tokyo, Japan: Waseda University. laurenceanthony.net/software. [24.09.2021]

Barsalou, Lawrence W. (1992): „Frames, Concepts, and Conceptual Fields“. In: Lehrer, Adrienne/Feder Kittay, Eva (eds.): Frames, fields and contrasts. New Essays in Semantics and Lexical Organisation. Hillsdale, Erlbaum: 21-74.

Berkeleyer FrameNet (2021): FrameNet project. framenet.icsi.berkeley.edu/fndrupal/ [12.10.2021].

Blackall, Eric A. (1966): Die Entwicklung des Dt. zur Literatursprache 1700-1775. Mit einem Bericht über neue Forschungsergebnisse 1955-1964 von Dieter Kimpel. Stuttgart: Metzler.

Boas, Hans C./Fuchs, Katrin (2018): „Zum Einfluss des Standarddeutschen auf das Texasdeutsche im 19. und 20. Jahrhundert: Empirische und methodologische Probleme“. In: Lenz, Alexandra N./Plewnia, Albrecht (eds.): Variation - Normen - Identitäten. Berlin/Boston, De Gruyter: 283-303.

\footnotetext{
35 Dudens Vollständiges Orthographisches Wörterbuch der deutschen Sprache wurde im Jahr 1880 in der ersten Auflage veröffentlich, die allgemeingültige Standardorthographie wurde 1903 an deutschen Schulen und in deutschen Behörden Pflicht (cf. Boas/Fuchs 2018: 291).
} 
Bodmer, Johann Jakob (1768): Die Grundsätze der deutschen Sprache. Oder: Von den Bestandtheilen derselben und von dem Redesatze Oder: Von den Bestandtheilen derselben und von dem Redesatze. Zürich: Orell, Geßner und Comp.

Busse, Dietrich (2012): Frame-Semantik. Ein Kompendium. Berlin/Boston: De Gruyter.

Busse, Dietrich (2018a): „Diskurs und Wissensrahmen“. In: Warnke, Ingo H. (ed.): Handbuch Diskurs. Boston, De Gruyter: 3-29.

Busse, Dietrich (2018b): „Überlegungen zu einem integrativen Frame-Modell. Elemente, Ebenen, Aspekte“. In: Ziem, Alexander/Inderelst, Lars/Wulf, Detmer (eds.): Frames interdisziplinär: Modelle, Anwendungsfelder, Methoden. Düsseldorf, dup: 69-92.

Busse, Dietrich/Teubert, Wolfgang (1994): „Ist Diskurs ein sprachwissenschaftliches Objekt? Zur Methodenfrage der Historischen Semantik“. In: Teubert, Wolfgang/Busse, Dietrich/Hermanns, Fritz (eds.): Begriffsgeschichte und Diskursgeschichte. Methodenfragen und Forschungsergebnisse der historischen Semantik. Opladen, Westdeutscher Verlag: 10-28.

Dornblüth, Augustin (1755): Observationes oder gründliche Anmerckungen über die Art und Weise eine gute Ubersetzung besonders in die teutsche Sprach zu machen ... Nebst einer ... Critic über Herrn Gottschedens sogenannte Redekunst, und teutsche Grammatic ... Augsburg: Rieger.

Elspaß, Stephan/Dürscheid, Christa/Ziegler, Arne (2017): „Zur grammatischen Pluriarealität der deutschen Gebrauchsstandards - oder: Über die Grenzen des Plurizentrizitätsbegriffs“. In: Zeitschrift für deutsche Philologie. Thematisches Sonderheft: Das Deutsche als plurizentrische Sprache. Ansprüche - Ergebnisse - Perspektiven. Herausgegeben von Sieburg, Heinz/ Solms, Hans-Joachim: 1-26. researchgate.net/publication/322273348 [14.05.2021].

Faulstich, Katja (2008): Konzepte des Hochdeutschen. Der Sprachnormierungsdiskurs im 18. Jahrhundert. Berlin: De Gruyter.

Foucault, Michel (1981): Archäologie des Wissens. Frankfurt a. M.: Suhrkamp.

Frangk, Fabian (1531): Teutscher Sprach und Eygenschafft Orthographia, gerecht buchstäbig Teutsch zuschreiben. New Cantzlei ietz brauchiger gerechter Practick formliche Missiuen und Schrifften an iede Personen rechtmessig zu stellen auffs kürtzst begriffen. Franckfurt: Egenolph.

Fulda, Friedrich Carl (1778): Grundregeln der teutschen Sprache. Stuttgart: Mezler.

Gardt, Andreas (2007): „Diskursanalyse“. Aktueller theoretischer Ort und methodische Möglichkeiten“. In: Warnke, Ingo H. (ed.): Diskurslinguistik nach Foucault. Theorie und Gegenstände. Berlin/New York, De Gruyter: 28-52.

Gardt, Andreas (2017): „Zum Diskursbegriff“. Der Deutschunterricht 6/2017: 2-7.

German FrameNet (GFN) (2019): FrameNet des Deutschen. Analyse von Frames. gsw.phil.hhu.de/framenet/wp?id=157 [18.02.2021].

German FrameNet Projekt (2021): German FrameNet Projekt. gsw.phil.hhu.de/framenet/. [12.10.2021]

Gottsched, Johann Christoph (1748): Grundlegung einer Deutschen Sprachkunst. Nach den Mustern der besten Schriftsteller des vorigen und jetzigen Jahrhunderts abgefasset von Johann Christoph Gottscheden. Leipzig: Breitkopf.

Gottsched, Johann Christoph (1762): Vollständigere und Neuerläuterte Deutsche Sprachkunst. Nach den Mustern der besten Schriftsteller des vorigen und itzigen Jahrhunderts abgefasset, 
und bey dieser fünften Auflage merklich verbessert von Johann Christoph Gottscheden. Leipzig: Breitkopf.

Hundt, Markus (1992): Einstellungen gegenüber dialektal gefärbter Standardsprache. Eine empirische Untersuchung zum Bairischen, Hamburgischen, Pfälzischen und Schwäbischen. Stuttgart: Steiner.

Josten, Dirk (1976): Sprachvorbild und Sprachnorm im Urteil des 16. und 17. Jahrhunderts. Bern/Frankfurt a. M.: Lang.

Jürgens, Carolin (2015): Niederdeutsch im Wandel. Sprachgebrauchswandel und Sprachwahrnehmung in Hamburg. Hildesheim etc..: Olms.

Kolroß, Johannes (1530): Enchiridion: das ist, Handbuechlin tütscher Orthographi, hochtütsche sprach artlich zeschryben/vnd laesen/ sampt eynem Registerlin über die gantze Bibel/ wie man die Allegationes vnd Concordantias/so im Nüwen Testament naeben dem text vnnd sunst mit halben Latinischen worten verzeychnet/ Ouch wie man die Zifer und tütsche zaal verston soll. Durch Joannem Kolross/ tüdtsch Leermeystern zuo Basel. Basel: Wolff.

Lenz, Alexandra N. (2003): Struktur und Dynamik des Substandards. Eine Studie zum Westmitteldeutschen (Wittlich/Eifel). Stuttgart: Steiner.

Lönneker, Birte (2003): Konzeptframes und Relationen. Extraktion, Annotation und Analyse französischer Corpora aus dem World Wide Web. Berlin: AKA.

Müller, Johannes (1969): Quellenschriften und Geschichte des deutschsprachlichen Unterrichts bis zur Mitte des 16. Jahrhunderts. Darmstadt: Wissenschaftliche Buchgesellschaft.

Popowitsch, Johann Siegmund Valentin (1754): Die notwendigsten Anfangsgründe der teutschen Sprachkunst zum Gebrauche der österreichischen Schulen. Wien: Grundt.

Quasthoff, Uta (1998): „Stereotype in Alltagsargumentationen. Ein Beitrag zur Dynamisierung der Stereotypenforschung“. In: Heinemann, Margot (ed.): Sprachliche und soziale Stereotype. Frankfurt a. M., Lang: 47-72.

Rosenberg, Milton J./Hovland, Carl I. (1966): „Cognitive, affective, and behavioural components of attitudes“. In: Rosenberg, Milton J./Hovland, Carl I. (eds.): Attitude organization and change: An analysis of consistency among attitude components. New Haven, Yale University Press: 1-14.

Sauer, Verena (in Vorbereitung): Framesemantische Analyse historischer Spracheinstellungen des 16. bis 18. Jahrhunderts. (Habilitationsschrift).

Scharloth, Joachim (2005): Sprachnormen und Mentalitäten. Sprachbewusstseinsgeschichte in Deutschland im Zeitraum von 1766 und 1785. Tübingen: Niemeyer.

Schlosser, Horst Dieter (1985): „Sprachnorm und regionale Differenz im Rahmen der Kontroverse zwischen Gottsched und Bodmer/Breitinger“. In: Kimpel, Dieter (ed.): Mehrsprachigkeit in der deutschen Aufklärung. Hamburg, Meiner. 52-68.

Schröder, Ingrid (2019): „Sprachbiografie und Spracheinstellung. Niederdeutsch als Mittel der Identitätsstiftung in der Großstadt? In: Eichinger, Ludwig M./Plewnia, Albrecht (eds.): Neues vom heutigen Deutsch. Empirisch - methodisch - theoretisch. Berlin/Boston, De Gruyter: 99-120.

Thiele, Martina (2015): Medien und Stereotype. Konturen eines Forschungsfeldes. Bielefeld: transcript.

von Polenz, Peter (1999): Deutsche Sprachgeschichte vom Spätmittelalter bis zur Gegenwart. Band III. 19. und 20. Jahrhundert. Berlin: De Gruyter. 
von Polenz, Peter (2009): Geschichte der deutschen Sprache. Berlin/New York: De Gruyter. von Polenz, Peter (2013): Deutsche Sprachgeschichte vom Spätmittelalter bis zur Gegenwart.

Band II. 17. und 18. Jahrhundert. Berlin/Boston: De Gruyter.

Ziem, Alexander (2005): Frame-Semantik und Diskursanalyse. Zur Verwandtschaft zweier Wissensanalysen. https://www.phil-fak.uni-duesseldorf.de/fileadmin/Redaktion/Institute/ Germanistik/Konstruktionsgrammatik/ZiemFrames_Diskurs.pdf [12.10.2021].

Ziem, Alexander (2008): Frames und sprachliches Wissen. Kognitive Aspekte der semantischen Kompetenz. Berlin/New York: De Gryuter.

Ziem, Alexander (2014): Frames of Understanding in Text and Discourse: Theoretical Foundations and Descriptive Applications. Amsterdam/Philadelphia: Benjamins.

Ziem, Alexander (2018): „Frames interdisziplinär: zur Einleitung.“ In: Ziem, Alexander/Inderelst, Lars/Wulf, Detmer (eds.): Proceedings of the Interdisciplinary Workshop „,Frame-Theorien im Vergleich: Modelle, Anwendungsfelder, Methoden “. Düsseldorf, Düsseldorf University Press: 7-22.

\section{Anhang}

Frame Hochdeutsch bei Gottsched: Vorrede zu seiner Sprachkunst (1748)

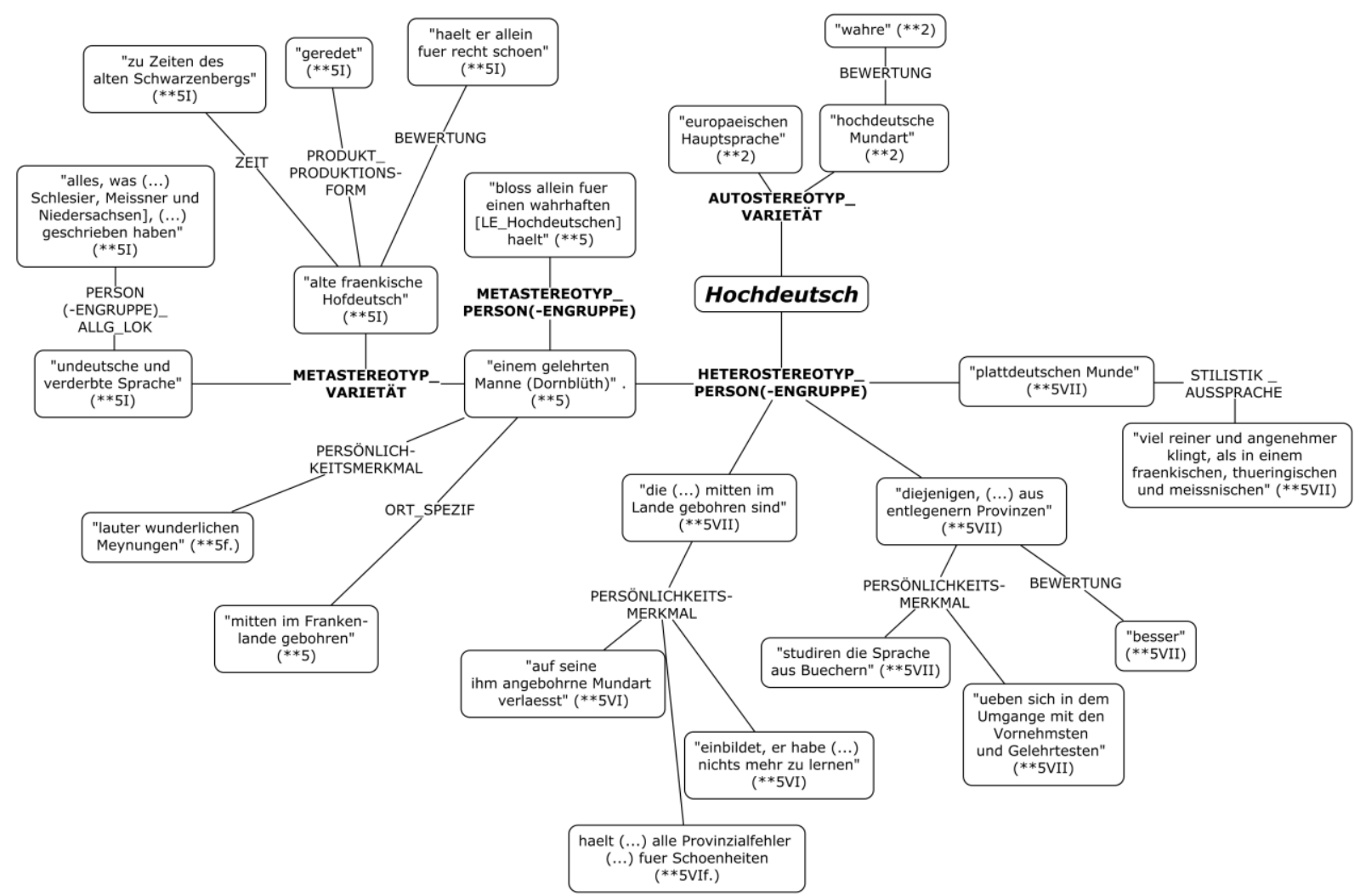

Abbildung 4: Frame Hochdeutsch bei Gottsched: Vorrede zu seiner Sprachkunst (1748) 


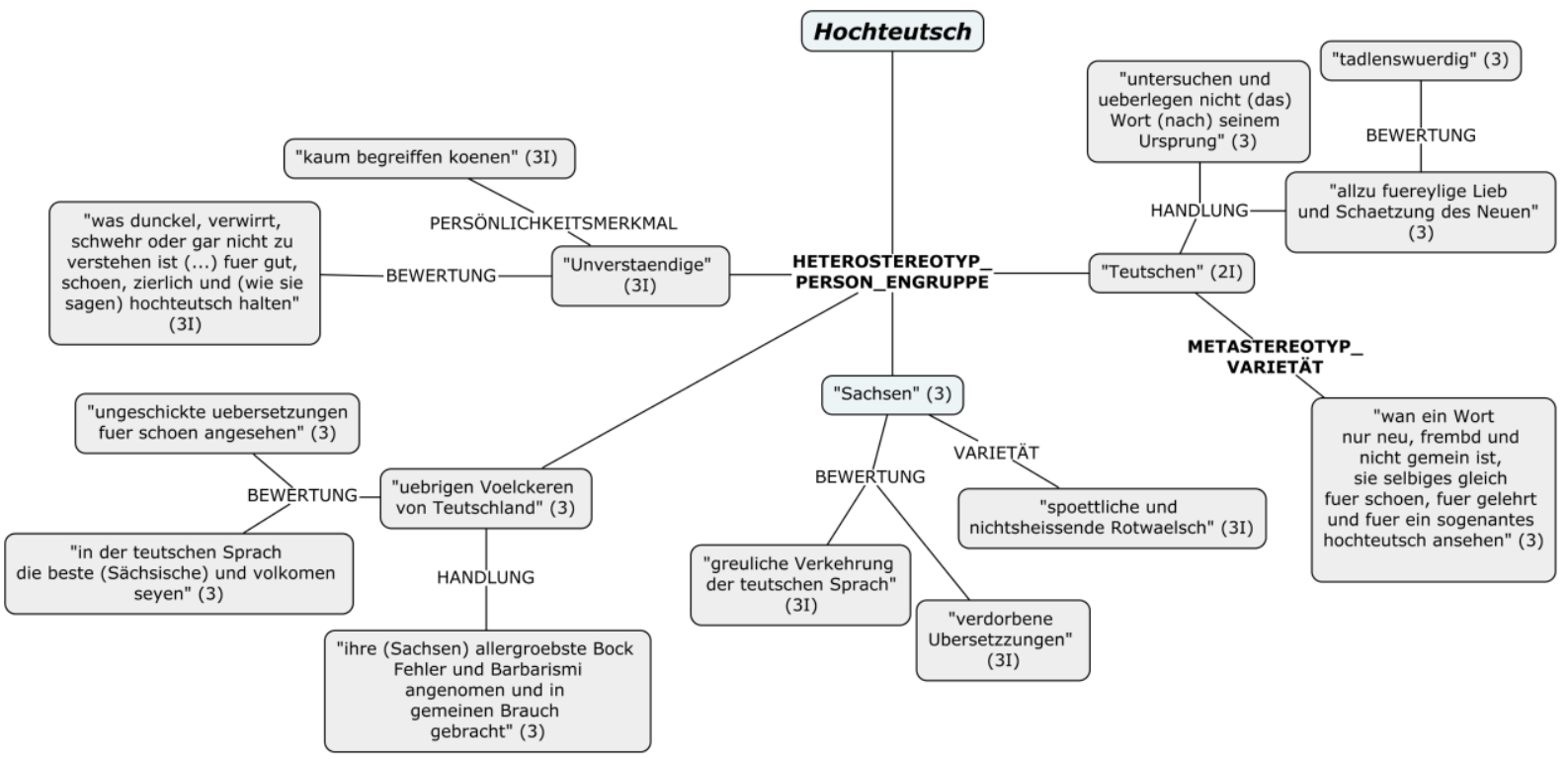

Abbildung 5: Frame Hochteutsch bei Dornblüth: Vorrede zu seinen Observationes (1755)

Frame Hochdeutsch bei Popowitsch: Vorrede zu seiner Sprachkunst (1754)

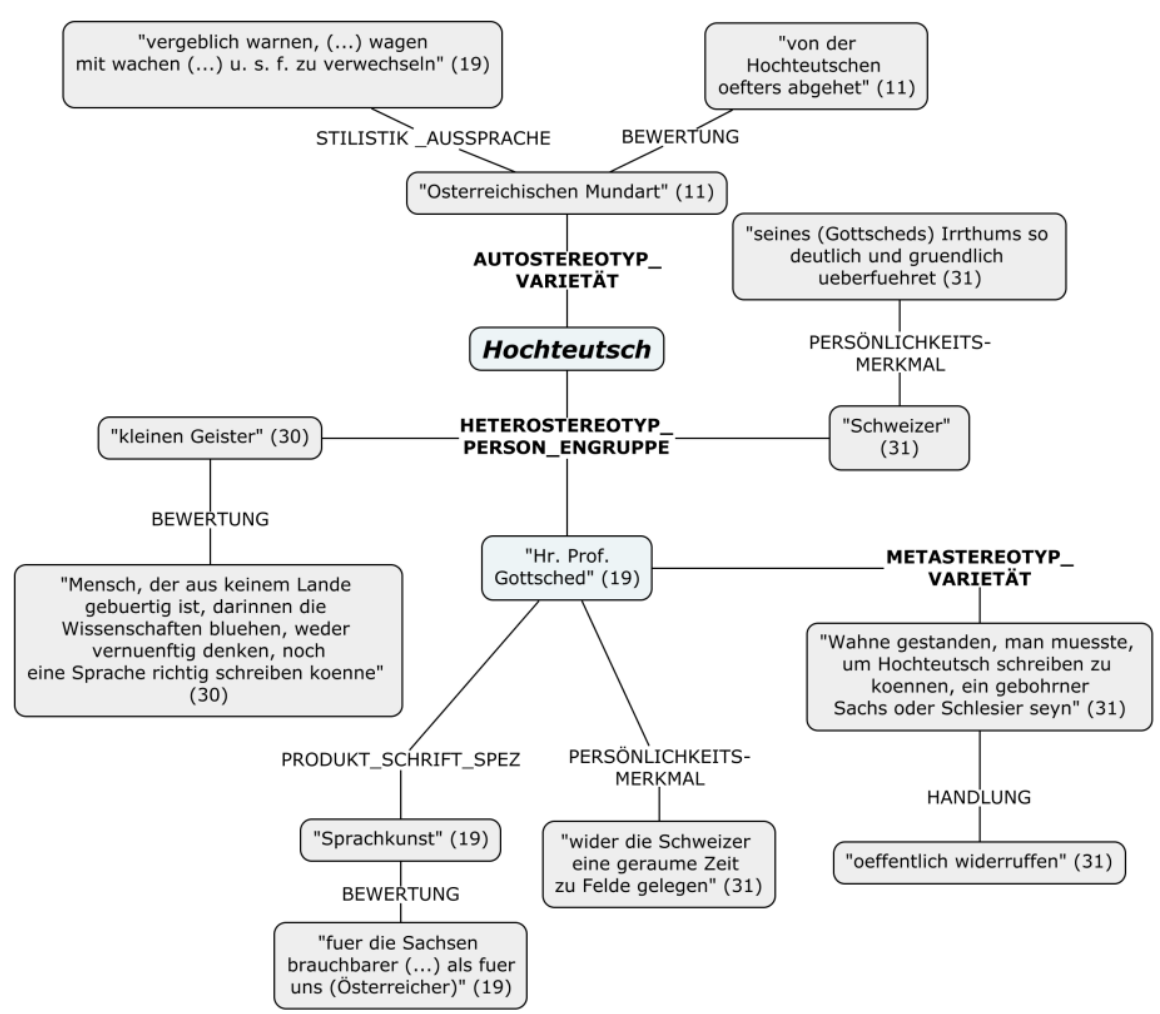

Abbildung 6: Frame Hochteutsch bei Popowitsch: Vorrede zu seiner Sprachkunst (1754) 
Frame Hochdeutsch bei Bodmer: Vorrede zu seinen Grundsätzen (1768)

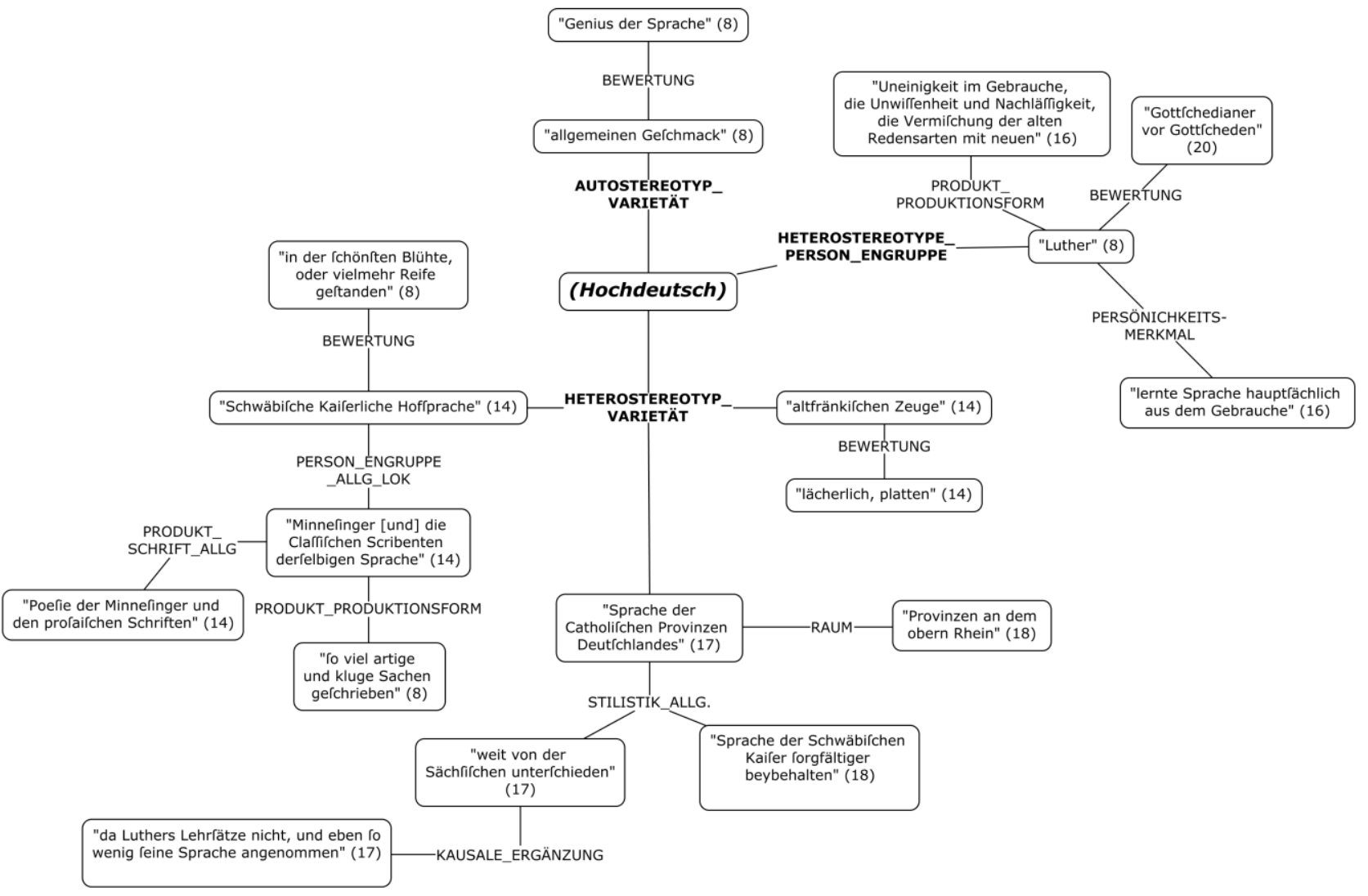

Abbildung 7: Frame Hochdeutsch bei Bodmer: Vorrede zu seinen Grundsätzen der deutschen Sprache (1768)

Frame Hochdeutsch bei Antesperg: Vorrede zu seiner Grammatick (1749)

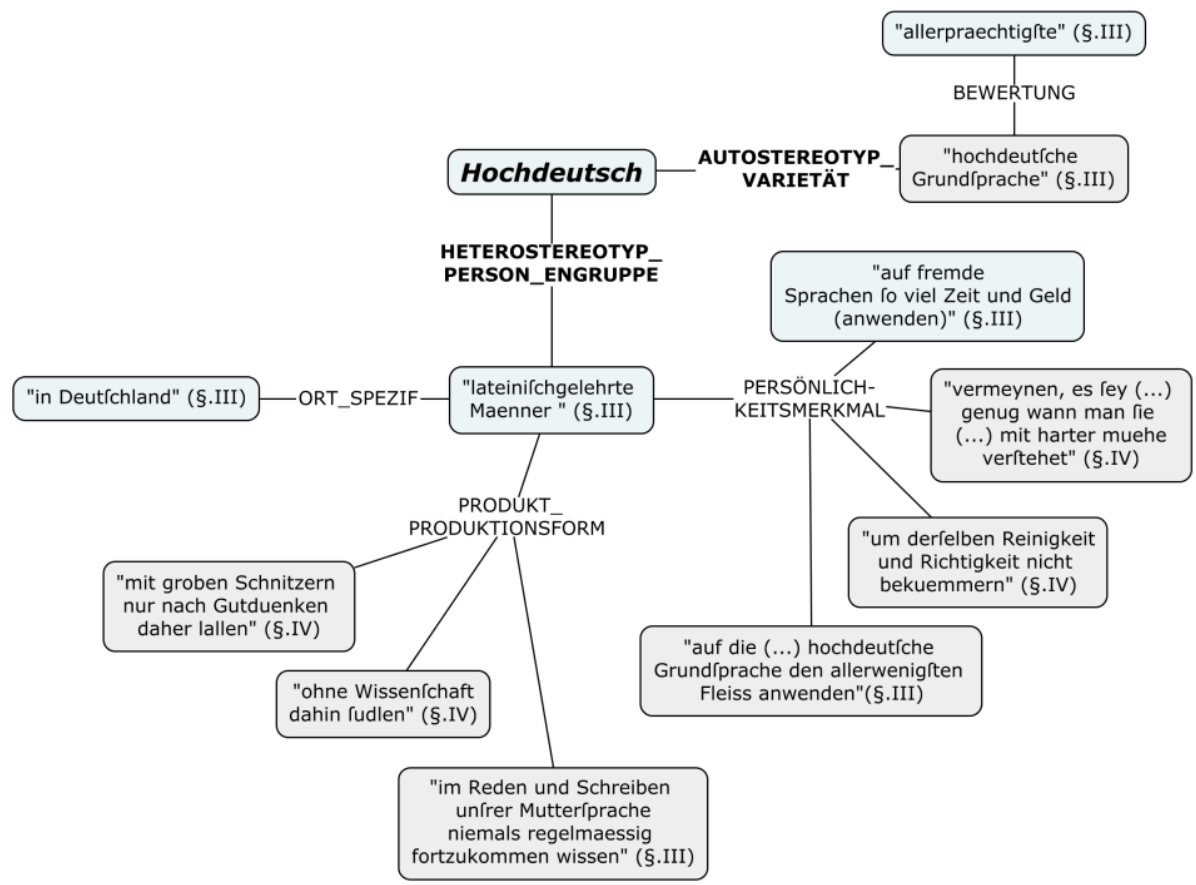

Abbildung 8: Frame Hochdeutsch bei Antesperg: Vorrede zu seiner Grammatick (1749) 
Frame Hochdeutsch bei Aichinger: Vorrede zu seiner Sprachlehre (1754)

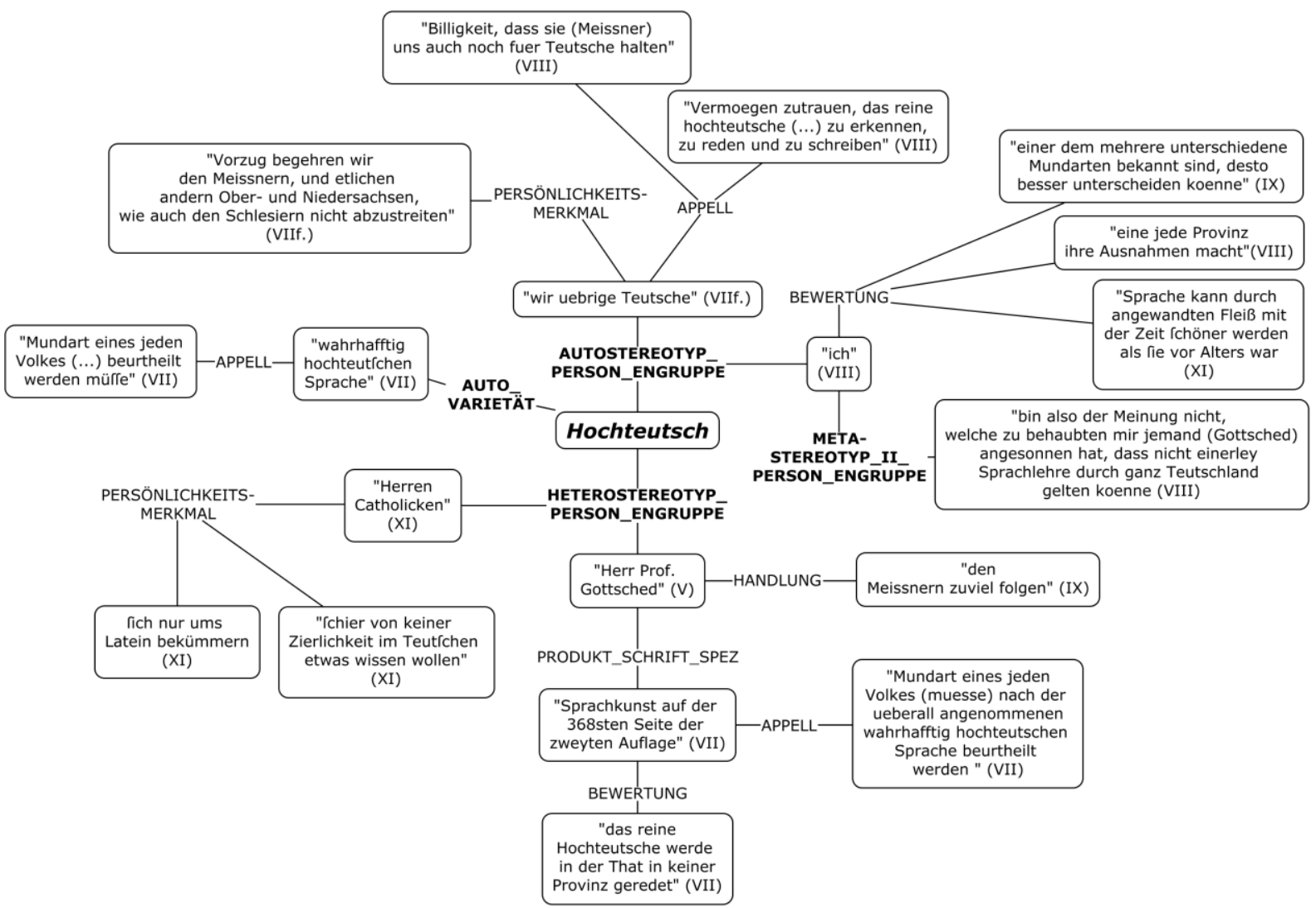

Abbildung 9: Frame Hochteutsch bei Aichinger: Vorrede zu seiner Sprachlehre (1754) 